\title{
On the singularity structure of Kahan discretizations of a class of quadratic vector fields
}

\section{René Zander ${ }^{1}$}

Received: 9 March 2020 / Accepted: 20 May 2021 / Published online: 30 June 2021

(c) The Author(s) 2021

\begin{abstract}
We discuss the singularity structure of Kahan discretizations of a class of quadratic vector fields and provide a classification of the parameter values such that the corresponding Kahan map is integrable, in particular, admits an invariant pencil of elliptic curves.
\end{abstract}

Keywords Birational map · Integrable map $\cdot$ Elliptic curve $\cdot$ Elliptic pencil

Mathematics Subject Classification 37J70 • 14H70 · 14E05

\section{Introduction}

The Kahan discretization scheme was introduced in the unpublished notes [13] as a method applicable to any system of ordinary differential equations in $\mathbb{R}^{n}$ with a quadratic vector field

$$
f(\mathbf{x})=Q(\mathbf{x})+B \mathbf{x}+c, \quad \mathbf{x} \in \mathbb{R}^{n},
$$

where each component of $Q: \mathbb{R}^{n} \rightarrow \mathbb{R}^{n}$ is a quadratic form, while $B \in \mathbb{R}^{n \times n}$ and $c \in \mathbb{R}^{n}$. Kahan's discretization reads as

$$
\frac{\widetilde{\mathbf{x}}-\mathbf{x}}{2 \varepsilon}=Q(\mathbf{x}, \widetilde{\mathbf{x}})+\frac{1}{2} B(\mathbf{x}+\widetilde{\mathbf{x}})+c,
$$

This research is supported by the DFG Collaborative Research Center TRR 109 "Discretization in Geometry and Dynamics".

$凶$ René Zander

zander@math.tu-berlin.de

1 Institut für Mathematik, MA 7-1, Technische Universität Berlin, Str. des 17. Juni 136, 10623 Berlin, Germany 
where

$$
Q(\mathbf{x}, \widetilde{\mathbf{x}})=\frac{1}{2}(Q(\mathbf{x}+\widetilde{\mathbf{x}})-Q(\mathbf{x})-Q(\widetilde{\mathbf{x}}))
$$

is the symmetric bilinear form corresponding to the quadratic form $Q$. Equation (1) is linear with respect to $\mathbf{x}$ and therefore defines a rational map $\widetilde{\mathbf{x}}=\phi_{\varepsilon}(\mathbf{x})$. Since Eq. (1) remains invariant under the interchange $\mathbf{x} \leftrightarrow \widetilde{\mathbf{x}}$ with the simultaneous sign inversion $\varepsilon \mapsto-\varepsilon$, one has the reversibility property $\phi_{\varepsilon}^{-1}(\mathbf{x})=\phi_{-\varepsilon}(\mathbf{x})$. In particular, the map $\phi_{\varepsilon}$ is birational.

In this paper, we consider the class of two-dimensional quadratic differential equations

$$
\left(\begin{array}{c}
\dot{x} \\
\dot{y}
\end{array}\right)=\ell_{1}^{1-\gamma_{1}}(x, y) \ell_{2}^{1-\gamma_{2}}(x, y) \ell_{3}^{1-\gamma_{3}}(x, y) J \nabla H(x, y),
$$

where

$$
H(x, y)=\ell_{1}^{\gamma_{1}}(x, y) \ell_{2}^{\gamma_{2}}(x, y) \ell_{3}^{\gamma_{3}}(x, y)
$$

and

$$
\ell_{i}(x, y)=a_{i} x+b_{i} y
$$

are linear forms, with $a_{i}, b_{i} \in \mathbb{C}, J=\left(\begin{array}{cc}0 & 1 \\ -1 & 0\end{array}\right)$ and $\gamma_{1}, \gamma_{2}, \gamma_{3} \in \mathbb{R} \backslash\{0\}$.

Integrability of the Kahan maps $\phi: \mathbb{C}^{2} \rightarrow \mathbb{C}^{2}$ was established for several cases of parameters $\left(\gamma_{1}, \gamma_{2}, \gamma_{3}\right)$ : If $\left(\gamma_{1}, \gamma_{2}, \gamma_{3}\right)=(1,1,1)$, then $(2)$ is a canonical Hamiltonian system on $\mathbb{R}^{2}$ with homogeneous cubic Hamiltonian. For such systems, a rational integral for the Kahan map $\phi$ was found in $[4,17]$. The Kahan maps for the cases $\left(\gamma_{1}, \gamma_{2}, \gamma_{3}\right)=(1,1,2)$ and $\left(\gamma_{1}, \gamma_{2}, \gamma_{3}\right)=(1,2,3)$ were treated in [6,17,20]. In all three cases, the level sets of the integral for both the continuous time system and the Kahan discretization have genus 1 . If $\left(\gamma_{1}, \gamma_{2}, \gamma_{3}\right)=(1,1,0)$, then (2) is a Hamiltonian vector field on $\mathbb{R}^{2}$ with linear Poisson tensor and homogeneous quadratic Hamiltonian. In this case, a rational integral for the Kahan map $\phi$ was found in [5]. The level sets of the integral have genus 0 .

In this paper, we study the singularity structure of the Kahan discretization as a birational quadratic map $\phi: \mathbb{C P}^{2} \rightarrow \mathbb{C P}^{2}$. Based on general classification results by Diller and Favre [9], we provide the following classification for the Kahan map $\phi$ of (2) depending on the values of the parameters $\left(\gamma_{1}, \gamma_{2}, \gamma_{3}\right)$ :

Theorem 1.1 Let $\phi: \mathbb{C P}^{2} \rightarrow \mathbb{C P}^{2}$ be the Kahan map of (2). The sequence of degrees $d(m)$ of iterates $\phi^{m}$ grows exponentially, so that the map $\phi$ is non-integrable, except for the following cases:

(i) If $\left(\gamma_{1}, \gamma_{2}, \gamma_{3}\right)=(1,1,1),(1,1,2),(1,2,3)$, the sequence $d(m)$ of degrees grows quadratically. The map $\phi$ admits an invariant pencil of elliptic curves. The degree of a generic curve of the pencil is 3, 4, 6, respectively. 
(ii) If $\left(\gamma_{1}, \gamma_{2}, \gamma_{3}\right)=(1,1,0)$ or $\left(\gamma_{1}, \gamma_{2}, \gamma_{3}\right)=(\alpha, 1,-1), \alpha \in \mathbb{R} \backslash \mathbb{Z} \cup\{0\}$, the sequence of degrees $d(m)$ grows linearly. The map $\phi$ admits an invariant pencil of rational curves.

(iii) If $\left(\gamma_{1}, \gamma_{2}, \gamma_{3}\right)=(n, 1,-1), n \in \mathbb{N}$, the sequence of degrees $d(m)$ is bounded. Here, $\left(\gamma_{1}, \gamma_{2}, \gamma_{3}\right)$ are fixed up to permutation and multiplication by $\lambda \in \mathbb{R} \backslash\{0\}$.

Some of the integrable cases are discussed in further detail in Sects. 4, 5, 6, 7 and 8.

\section{Preliminary results}

\subsection{Birational maps of surfaces}

Definition 2.1 Let $\phi$ be a birational map of a smooth projective surface $X$. The dynamical degree of the map $\phi$ is defined as

$$
\lambda_{1}=\lim _{m \rightarrow \infty}\left\|\left(\phi^{m}\right)^{*}\right\|^{1 / m},
$$

where $\left(\phi^{m}\right)^{*}$ denote the induced pullback maps on the Picard group Pic $(X)$.

Diller and Favre provide the following classification for birational maps with $\lambda_{1}=1$ :

Theorem 2.2 (Diller, Favre [9, Theorem 0.2]) Let $\phi: X \rightarrow X$ be a birational map of a smooth projective surface with $\lambda_{1}=1$. Up to birational conjugacy, exactly one of the following holds:

(i) The sequence $\left\|\left(\phi^{m}\right)^{*}\right\|$ is bounded, and $\phi^{m}$ is an automorphism isotopic to the identity for some $m$.

(ii) The sequence $\left\|\left(\phi^{m}\right)^{*}\right\|$ grows linearly, and $\phi$ preserves a rational fibration. In this case, $\phi$ cannot be conjugated to an automorphism.

(iii) The sequence $\left\|\left(\phi^{m}\right)^{*}\right\|$ grows quadratically, and $\phi$ is an automorphism preserving an elliptic fibration.

One says that $\phi: X \rightarrow X$ is analytically stable (AS) if $\left(\phi^{*}\right)^{m}=\left(\phi^{m}\right)^{*}$ on $\operatorname{Pic}(X)$. This relates the dynamical degree $\lambda_{1}$ to the spectral radius of the induced pullback $\phi^{*}: \operatorname{Pic}(X) \rightarrow \operatorname{Pic}(X)$. Equivalently, analytic stability is characterized by the condition that there is no curve $V \subset X$ such that $\phi^{n}(V) \in \mathcal{J}(\phi)$ for some integer $n \geqslant 0$, where $\mathcal{J}(\phi)$ is the indeterminacy set of $\phi$ (see [9, Theorem 1.14]). Therefore, the notion of analytic stability is closely related to singularity confinement (see [15]). Indeed, a singularity confinement pattern for a map $\phi: X \rightarrow X$ involves a curve $V \subset X$ such that $\phi(V)=P$ is a point (so that $P \in \mathcal{J}\left(\phi^{-1}\right)$ ) and $\phi^{n-1}(P) \in \mathcal{J}(\phi)$, so that $\phi^{n}(P$ ) is a curve again for some positive integer $n \in \mathbb{N}$. Such a singularity confinement pattern can be resolved by blowing up the orbit of $P$. Upon resolving all singularity confinement patterns, one lifts $\phi$ to an AS map $\widetilde{\phi}: X^{\prime} \rightarrow X^{\prime}$.

Diller and Favre showed that for any birational map $\phi: X \rightarrow X$ of a smooth projective surface we can construct by a finite number of successive blow-ups a surface $X^{\prime}$ such that $\phi$ lifts to an analytically stable birational map $\widetilde{\phi}: X^{\prime} \rightarrow X^{\prime}$ (see $[9$, Theorem 0.1]). 


\subsection{Birational quadratic maps of $\mathbb{C P}^{2}$}

As shown, e.g., in [7], every quadratic birational map $\phi: \mathbb{C P}^{2} \rightarrow \mathbb{C P}^{2}$ can be represented as $\phi=A_{1} \circ q_{i} \circ A_{2}$, where $A_{1}, A_{2}$ are linear projective transformations of $\mathbb{C P}^{2}$ and $q_{i}$ is one of the three standard quadratic involutions:

$$
\begin{aligned}
& q_{1}:[x, y, z] \rightarrow[y z, x z, x y], \\
& q_{2}:[x, y, z] \rightarrow\left[x z, y z, x^{2}\right], \\
& q_{3}:[x, y, z] \rightarrow\left[x^{2}, x y, y^{2}+x z\right] .
\end{aligned}
$$

In these three cases, the indeterminacy set $\mathcal{J}(\phi)$ consists of three, respectively two, one (distinct) singularities. The last two cases correspond to a coalescence of singularities. Therefore, the first case is the generic one.

In the present work, we only consider the first case: $\phi=A_{1} \circ q_{1} \circ A_{2}$. In this case, $\mathcal{J}(\phi)=\left\{B_{+}^{(1)}, B_{+}^{(2)}, B_{+}^{(3)}\right\}$ consists of three distinct points. Let $L_{-}^{(1)}$ denote the line through $B_{+}^{(2)}, B_{+}^{(3)}$, and similarly for permutations of the indices 1,2,3. (We have, e.g., $B_{+}^{(1)}=L_{-}^{(2)} \cap L_{-}^{(3)}$.) These lines are exceptional in the sense that they are blown down by $\phi$ to points: $\phi\left(L_{-}^{(i)}\right)=B_{-}^{(i)}$. The inverse map is also quadratic with set of indeterminacy points $\mathcal{J}\left(\phi^{-1}\right)=\left\{B_{-}^{(1)}, B_{-}^{(2)}, B_{-}^{(3)}\right\}$.

Suppose that the map admits $s$ singularity confinement patterns, $0 \leqslant s \leqslant 3$. That means there are positive integers $n_{1}, \ldots, n_{s} \in \mathbb{N}$ and $\left(\sigma_{1}, \ldots, \sigma_{s}\right)$ such that $\phi^{n_{i}-1}\left(B_{-}^{(i)}\right)=B_{+}^{\left(\sigma_{i}\right)}$ for $i=1, \ldots, s$. We assume that the $n_{i}$ are taken to be minimal and, for simplicity, we also assume that $\phi^{k}\left(B_{-}^{(i)}\right) \neq \phi^{l}\left(B_{-}^{(j)}\right)$ for any $k, l \geqslant 0$ and $i \neq j$. As shown by Bedford and Kim [2], one can resolve the singularity confinement patterns by blowing up the finite sequences $B_{-}^{(i)}, \phi\left(B_{-}^{(i)}\right), \ldots, \phi^{n_{i}-1}\left(B_{-}^{(i)}\right)$. Those sequences are also called singular orbits. In this paper, we only encounter the situation that the orbits of different $B_{-}^{(i)}$ are disjoint. As shown in [2], one can adjust the procedure to the more general situation.

On the blow-up surface $X$, the lifted map $\widetilde{\phi}: X \rightarrow X$ is AS, and is an automorphism if and only if $s=3$. The $s$-tuples $\left(n_{1}, \ldots, n_{s}\right),\left(\sigma_{1}, \ldots, \sigma_{s}\right)$ are called orbit data associated to $\phi$. We say that the map $\phi$ realizes the orbit data $\left(n_{1}, \ldots, n_{s}\right),\left(\sigma_{1}, \ldots, \sigma_{s}\right)$.

Let $\mathcal{H} \in \operatorname{Pic}(X)$ be the pullback of the divisor class of a generic line in $\mathbb{C P}^{2}$. Let $\mathcal{E}_{i, n} \in \operatorname{Pic}(X)$, for $i \leqslant s$ and $0 \leqslant n \leqslant n_{i}-1$, be the divisor class of the exceptional divisor associated to the blow-up of the point $\phi^{n}\left(B_{-}^{(i)}\right)$. Then $\mathcal{H}$ and $\varepsilon_{i, n}$ give a basis for $\operatorname{Pic}(X)$, i.e.,

$$
\operatorname{Pic}(X)=\mathbb{Z} \mathcal{H} \bigoplus_{i=1}^{3} \bigoplus_{n=0}^{n_{i}-1} \mathbb{Z} \varepsilon_{i, n}
$$

that is orthogonal with respect to the intersection product, $(\cdot, \cdot)$ : $\operatorname{Pic}(X) \times \operatorname{Pic}(X) \rightarrow$ $\mathbb{Z}$, and is normalized by $(\mathcal{H}, \mathcal{H})=1$ and $\left(\mathcal{E}_{i, n}, \mathcal{E}_{i, n}\right)=-1$. The rank of the Picard group is $\sum n_{i}+1$. 
The induced pullback $\widetilde{\phi}^{*}: \operatorname{Pic}(X) \rightarrow \operatorname{Pic}(X)$ is determined by (see Bedford, Kim [2], and Diller [8])

$$
\begin{aligned}
\mathcal{H} & \mapsto 2 \mathcal{H}-\sum_{j \leqslant s} \mathcal{E}_{j, n_{j}-1}, \\
\mathcal{E}_{i, 0} & \mapsto \mathcal{H}-\sum_{\substack{j \leqslant s \\
\sigma_{j} \neq i}} \mathcal{E}_{j, n_{j}-1}, \quad i \leqslant s, \\
\mathcal{E}_{i, n} & \mapsto \mathcal{E}_{i, n-1}, \quad i \leqslant s, \quad 1 \leqslant n \leqslant n_{i}-1 .
\end{aligned}
$$

The induced pushforward $\widetilde{\phi}_{*}: \operatorname{Pic}(X) \rightarrow \operatorname{Pic}(X)$ is determined by

$$
\begin{aligned}
\mathcal{H} & \mapsto 2 \mathcal{H}-\sum_{j \leqslant s} \mathcal{E}_{j, n_{j}-1}, \\
\mathcal{E}_{i, 0} & \mapsto \mathcal{H}-\sum_{\substack{j \leqslant s \\
\sigma_{j} \neq i}} \mathcal{E}_{j, n_{j}-1}, \quad i \leqslant s, \\
\mathcal{E}_{i, n} & \mapsto \mathcal{E}_{i, n-1}, \quad i \leqslant s, \quad 1 \leqslant n \leqslant n_{i}-1 .
\end{aligned}
$$

The maps $\widetilde{\phi}^{*}, \widetilde{\phi}_{*}$ are adjoint with respect to the intersection product (see [9, Proposition 1.1]), i.e., $\left(\widetilde{\phi}^{*} A, B\right)=\left(A, \widetilde{\phi}_{*} B\right)$ for all $A, B \in \operatorname{Pic}(X)$.

Bedford and Kim computed the characteristic polynomial $\chi(\lambda)=\operatorname{det}\left(\widetilde{\phi}^{*}-\lambda\right.$ id $)$ explicitly for any given orbit data (see [2, Theorem 3.3]).

Let $C(m)=\left(\widetilde{\phi}^{*}\right)^{m}(\mathcal{H}) \in \operatorname{Pic}(X)$ be the class of the $m$ th iterate of a generic line. Set

$$
d(m)=(C(m), \mathcal{H})
$$

so that $d(m)$ is the algebraic degree of the $m$ th iterate of the map $\phi$. Set

$$
\mu_{i}(m+j)=\left(C(m), \mathcal{E}_{i, j}\right), \quad i \leqslant s, \quad 0 \leqslant j \leqslant n_{i}-1 .
$$

The expression on the right-hand side indeed depends on $i$ and $m+j$ only: using that the maps $\widetilde{\phi}^{*}, \widetilde{\phi}_{*}$ are adjoint with respect to the intersection product and the relations (4), we find

$$
\left(C(m), \mathcal{E}_{i, j}\right)=\left(C(m), \widetilde{\phi}_{*} \varepsilon_{i, j-1}\right)=\left(\widetilde{\phi}^{*} C(m), \mathcal{E}_{i, j-1}\right)=\left(C(m+1), \varepsilon_{i, j-1}\right) .
$$

In particular, $\mu_{i}(m)=\left(C(m), \mathcal{E}_{i, 0}\right)$ can be interpreted as the multiplicity of $B_{-}^{(i)}$ on the $m$ th iterate of a generic line.

The sequence of degrees $d(m)$ of iterates of the map $\phi$ satisfies a system of linear recurrence relations.

Theorem 2.3 (Recurrence relations) Let $\phi$ be a birational map of $\mathbb{C P}^{2}$ with three distinct indeterminacy points, and with associated orbit data $\left(n_{1}, \ldots, n_{s}\right),\left(\sigma_{1}, \ldots, \sigma_{s}\right)$. 
The degree of iterates $d(m)$ satisfies the system of recurrence relations

$$
\left\{\begin{array}{l}
d(m+1)=2 d(m)-\sum_{j \leqslant s} \mu_{j}(m), \\
\mu_{i}\left(m+n_{i}\right)=d(m)-\sum_{\substack{j \leqslant s \\
j \neq \sigma_{i}}} \mu_{j}(m), \quad i \leqslant s,
\end{array}\right.
$$

with initial conditions $d(0)=1$ and $\mu_{i}(m)=0$, for $i \leqslant s$ and $m=0, \ldots, n_{i}-1$.

Proof With (5), (6) we find that

$$
C(m)=d(m) \mathcal{H}-\sum_{i \leqslant s} \sum_{j=0}^{n_{i}-1} \mu_{i}(m+j) \mathcal{E}_{i, j} .
$$

With relations (3) we compute the pullback

$$
\begin{aligned}
& \widetilde{\phi}^{*} C(m) \\
& =d(m)\left(2 \mathcal{H}-\sum_{i \leqslant s} \mathcal{E}_{i, n_{i}-1}\right) \\
& \quad-\sum_{i \leqslant s}\left(\sum_{j=1}^{n_{i}-1} \mu_{i}(m+j) \mathcal{E}_{i, j-1}+\mu_{i}(m)\left(\mathcal{H}-\sum_{\substack{j \leqslant s \\
\sigma_{j} \neq i}} \mathcal{E}_{j, n_{j}-1}\right)\right) .
\end{aligned}
$$

Then we find

$$
\begin{aligned}
& \left(\widetilde{\phi}^{*} C(m), \mathcal{H}\right)=2 d(m)-\sum_{j \leqslant s} \mu_{j}(m), \\
& \left(\widetilde{\phi}^{*} C(m), \mathcal{E}_{i, n_{i}-1}\right)=d(m)-\sum_{\substack{j \leqslant s \\
j \neq \sigma_{i}}} \mu_{j}(m), \quad i \leqslant s, \\
& \left(\widetilde{\phi}^{*} C(m), \varepsilon_{i, j}\right)=\mu_{i}(m+1+j), \quad i \leqslant s, \quad 0 \leqslant j \leqslant n_{i}-2 .
\end{aligned}
$$

Finally, with $C(m+1)=\widetilde{\phi}^{*} C(m)$, we obtain recurrence relations (7). The initial conditions are $d(0)=(\mathcal{H}, \mathcal{H})=1$ and $\mu_{i}(j)=\left(\mathcal{H}, \mathcal{E}_{i, j}\right)=0$, for $i \leqslant s$ and $0 \leqslant j \leqslant n_{i}-1$. This proves the claim.

Corollary 2.4 (Generating functions) Consider the generating functions $d(z), \mu_{i}(z)$ for the sequences from Theorem 2.3. They are rational functions which can be defined 
as solutions of functional equations (8) with initial conditions as in Theorem 2.3.

$$
\left\{\begin{array}{l}
\frac{1}{z}(d(z)-1)=2 d(z)-\sum_{j \leqslant s} \mu_{j}(z), \\
\frac{1}{z^{n_{i}}} \mu_{i}(z)=d(z)-\sum_{\substack{j \leqslant s \\
j \neq \sigma_{i}}} \mu_{j}(z), \quad i \leqslant s .
\end{array}\right.
$$

\section{The $\left(\gamma_{1}, \gamma_{2}, \gamma_{3}\right)$-class}

The class of quadratic differential equations we want to consider is a generalization of the two-dimensional reduced Nahm systems introduced in [11],

$$
\left\{\begin{array} { l } 
{ \dot { x } = x ^ { 2 } - y ^ { 2 } , } \\
{ \dot { y } = - 2 x y , }
\end{array} \quad \left\{\begin{array} { l } 
{ \dot { x } = 2 x ^ { 2 } - 1 2 y ^ { 2 } , } \\
{ \dot { y } = - 6 x y - 4 y ^ { 2 } , }
\end{array} \quad \left\{\begin{array}{l}
\dot{x}_{1}=2 x^{2}-y^{2}, \\
\dot{x}_{2}=-10 x y+y^{2}
\end{array}\right.\right.\right.
$$

Such systems can be explicitly integrated in terms of elliptic functions and they admit integrals of motion given respectively by

$$
\begin{aligned}
& H_{1}(x, y)=\frac{y}{3}\left(3 x^{2}-y^{2}\right), \\
& H_{2}(x, y)=y(2 x+3 y)(x-y)^{2}, \\
& H_{3}(x, y)=\frac{y}{6}(3 x-y)^{2}(4 x+y)^{3} .
\end{aligned}
$$

Note that the curves $\left\{H_{i}(x, y)=\lambda\right\}$ are of genus 1 . Systems (9) were discussed in [11] and discretized by means of the Kahan method in [17]. The integrability of the Kahan discretizations

$$
\begin{aligned}
& \left\{\begin{array}{l}
\tilde{x}-x=2 \varepsilon(\tilde{x} x-\tilde{y} y), \\
\tilde{y}-y=-2 \varepsilon(\tilde{x} y+x \tilde{y}),
\end{array}\right. \\
& \left\{\begin{array}{l}
\tilde{x}-x=\varepsilon(4 \tilde{x} x-24 \tilde{y} y), \\
\tilde{y}-y=-\varepsilon(6 \tilde{x} y+6 x \tilde{y}+8 \tilde{y} y),
\end{array}\right. \\
& \left\{\begin{array}{l}
\tilde{x}-x=\varepsilon(4 \tilde{x} x-2 \tilde{y} y), \\
\tilde{y}-y=\varepsilon(-10 \tilde{x} y-10 x \tilde{y}+2 \tilde{y} y),
\end{array}\right.
\end{aligned}
$$

was shown in [17]. They were studied in the context of minimization of rational elliptic surfaces in [3]. The following generalization of reduced Nahm systems was introduced in $[6,20]$. 
We use the notation $\mathbf{x}=(x, y) \in \mathbb{C}^{2}$. Consider the two-dimensional quadratic differential equations

$$
\begin{aligned}
\dot{\mathbf{x}} & =\ell_{1}^{1-\gamma_{1}}(\mathbf{x}) \ell_{2}^{1-\gamma_{2}}(\mathbf{x}) \ell_{3}^{1-\gamma_{3}}(\mathbf{x}) J \nabla H(\mathbf{x}), \\
& =\gamma_{1} \ell_{2}(\mathbf{x}) \ell_{3}(\mathbf{x}) J \nabla \ell_{1}+\gamma_{2} \ell_{1}(\mathbf{x}) \ell_{3}(\mathbf{x}) J \nabla \ell_{2}+\gamma_{3} \ell_{1}(\mathbf{x}) \ell_{2}(\mathbf{x}) J \nabla \ell_{3},
\end{aligned}
$$

where

$$
H(\mathbf{x})=\ell_{1}^{\gamma_{1}}(\mathbf{x}) \ell_{2}^{\gamma_{2}}(\mathbf{x}) \ell_{3}^{\gamma_{3}}(\mathbf{x})
$$

and

$$
\ell_{i}(x, y)=a_{i} x+b_{i} y
$$

are linear forms, with $a_{i}, b_{i} \in \mathbb{C}, J=\left(\begin{array}{cc}0 & 1 \\ -1 & 0\end{array}\right)$ and $\gamma_{1}, \gamma_{2}, \gamma_{3} \in \mathbb{R} \backslash\{0\}$. System (10) has the function (11) as an integral of motion and an invariant measure form

$$
\Omega(\mathbf{x})=\frac{\mathrm{d} x \wedge \mathrm{d} y}{\ell_{1}(\mathbf{x}) \ell_{2}(\mathbf{x}) \ell_{3}(\mathbf{x})} .
$$

The Kahan discretization of (10) reads

$$
\begin{aligned}
\widetilde{\mathbf{x}}-\mathbf{x}=\varepsilon \gamma_{1}\left(\ell_{2}(\mathbf{x})\right. & \left.\ell_{3}(\widetilde{\mathbf{x}})+\ell_{2}(\widetilde{\mathbf{x}}) \ell_{3}(\mathbf{x})\right) J \nabla \ell_{1} \\
& +\varepsilon \gamma_{2}\left(\ell_{1}(\mathbf{x}) \ell_{3}(\widetilde{\mathbf{x}})+\ell_{1}(\widetilde{\mathbf{x}}) \ell_{3}(\mathbf{x})\right) J \nabla \ell_{2} \\
& +\varepsilon \gamma_{3}\left(\ell_{1}(\mathbf{x}) \ell_{2}(\widetilde{\mathbf{x}})+\ell_{1}(\widetilde{\mathbf{x}}) \ell_{2}(\mathbf{x})\right) J \nabla \ell_{3} .
\end{aligned}
$$

It was shown in [20] that the Kahan map admits (12) as invariant measure form. Now, multiplying (13) from the left by the vectors $\nabla \ell_{i}^{\mathrm{T}}, i=1,2,3$, we obtain

$$
\begin{array}{r}
\ell_{1}(\widetilde{\mathbf{x}})-\ell_{1}(\mathbf{x})=\varepsilon d_{12} \gamma_{2}\left(\ell_{1}(\mathbf{x}) \ell_{3}(\widetilde{\mathbf{x}})+\ell_{1}(\widetilde{\mathbf{x}}) \ell_{3}(\mathbf{x})\right) \\
-\varepsilon d_{31} \gamma_{3}\left(\ell_{1}(\mathbf{x}) \ell_{2}(\widetilde{\mathbf{x}})+\ell_{1}(\widetilde{\mathbf{x}}) \ell_{2}(\mathbf{x})\right), \\
\ell_{2}(\widetilde{\mathbf{x}})-\ell_{2}(\mathbf{x})=\varepsilon d_{23} \gamma_{3}\left(\ell_{1}(\mathbf{x}) \ell_{2}(\widetilde{\mathbf{x}})+\ell_{1}(\widetilde{\mathbf{x}}) \ell_{2}(\mathbf{x})\right) \\
-\varepsilon d_{12} \gamma_{1}\left(\ell_{2}(\mathbf{x}) \ell_{3}(\widetilde{\mathbf{x}})+\ell_{2}(\widetilde{\mathbf{x}}) \ell_{3}(\mathbf{x})\right), \\
\ell_{3}(\widetilde{\mathbf{x}})-\ell_{3}(\mathbf{x})=\varepsilon d_{31} \gamma_{1}\left(\ell_{2}(\mathbf{x}) \ell_{3}(\widetilde{\mathbf{x}})+\ell_{2}(\widetilde{\mathbf{x}}) \ell_{3}(\mathbf{x})\right) \\
-\varepsilon d_{23} \gamma_{2}\left(\ell_{1}(\mathbf{x}) \ell_{3}(\widetilde{\mathbf{x}})+\ell_{1}(\widetilde{\mathbf{x}}) \ell_{3}(\mathbf{x})\right),
\end{array}
$$

where

$$
d_{i j}=a_{i} b_{j}-a_{j} b_{i}
$$

From equations (14) to (16) it follows that the Kahan map leaves the lines $\left\{\ell_{i}(\mathbf{x})=0\right\}$, $i=1,2,3$, invariant. 
Explicitly, the Kahan discretization of (10) as map $\phi_{+}: \mathbb{C P}^{2} \rightarrow \mathbb{C P}^{2}$ is as follows:

$$
\phi_{+}:[x, y, z] \rightarrow\left[x^{\prime}, y^{\prime}, z^{\prime}\right]
$$

with

$$
\begin{aligned}
& x^{\prime}=z x+\varepsilon A_{2}(x, y), \\
& y^{\prime}=z y-\varepsilon B_{2}(x, y), \\
& z^{\prime}=z^{2}+z \varepsilon C_{1}(x, y)-2 \varepsilon^{2} C_{2}(x, y),
\end{aligned}
$$

with homogeneous polynomials of $\operatorname{deg} \leqslant 2$

$$
\begin{aligned}
& A_{2}(x, y)=\sum_{(i, j, k)} \gamma_{i} \ell_{i}(x, y)\left(b_{k} \ell_{j}(x, y)+b_{j} \ell_{k}(x, y)\right), \\
& B_{2}(x, y)=\sum_{(i, j, k)} \gamma_{i} \ell_{i}(x, y)\left(a_{k} \ell_{j}(x, y)+a_{j} \ell_{k}(x, y)\right), \\
& C_{1}(x, y)=\sum_{(i, j, k)} \gamma_{i}\left(d_{i k} \ell_{j}(x, y)+d_{i j} \ell_{k}(x, y)\right), \\
& C_{2}(x, y)=\sum_{(i, j, k)} \gamma_{j} \gamma_{k} d_{j k}^{2} \ell_{i}^{2}(x, y),
\end{aligned}
$$

where $\sum_{(i, j, k)}$ denotes the sum over all cyclic permutations of $(i, j, k)$ of $(1,2,3)$.

The inverse $\phi_{-}: \mathbb{C P}^{2} \rightarrow \mathbb{C P}^{2}$ of the Kahan map (17) is obtained by replacing $\varepsilon$ with $-\varepsilon$.

Lemma 3.1 The following identities hold:

$$
\begin{aligned}
& A_{2}\left(-\lambda b_{i}, \lambda a_{i}\right)=-b_{i} d_{i j} d_{k i}\left(\gamma_{j}+\gamma_{k}\right) \lambda^{2}, \\
& B_{2}\left(-\lambda b_{i}, \lambda a_{i}\right)=-a_{i} d_{i j} d_{k i}\left(\gamma_{j}+\gamma_{k}\right) \lambda^{2}, \\
& C_{1}\left(-\lambda b_{i}, \lambda a_{i}\right)=-d_{i j} d_{k i}\left(2 \gamma_{i}-\gamma_{j}-\gamma_{k}\right) \lambda, \\
& C_{2}\left(-\lambda b_{i}, \lambda a_{i}\right)=\gamma_{i} d_{i j}^{2} d_{k i}^{2}\left(\gamma_{j}+\gamma_{k}\right) \lambda^{2},
\end{aligned}
$$

where $(i, j, k)$ is a cyclic permutation of $(1,2,3)$.

Proof This is the result of straightforward computations.

In the following, we assume that $d_{12}, d_{23}, d_{31} \neq 0$, i.e., that the lines $\left\{\ell_{i}(x, y)=0\right\}$ are pairwise distinct. Also, we consider $\mathbb{C}^{2}$ as affine part of $\mathbb{C P}^{2}$ consisting of the points $[x, y, z] \in \mathbb{C P}^{2}$ with $z \neq 0$. We identify the point $(x, y) \in \mathbb{C}^{2}$ with the point $[x, y, 1] \in \mathbb{C P}^{2}$.

Proposition 3.2 The singularities $B_{+}^{(i)}, i=1,2,3$, of the Kahan map $\phi_{+}$and $B_{-}^{(i)}$, $i=1,2,3$, of its inverse $\phi_{-}$are given by

$$
B_{ \pm}^{(i)}=\left[ \pm \frac{b_{i}}{\varepsilon d_{i j} d_{k i}}, \mp \frac{a_{i}}{\varepsilon d_{i j} d_{k i}}, \gamma_{j}+\gamma_{k}\right] \text {, }
$$


where $(i, j, k)$ is a cyclic permutation of $(1,2,3)$. Let $\mathcal{L}_{\mp}^{(i)}$ denote the line through the points $B_{ \pm}^{(j)}, B_{ \pm}^{(k)}$. Then we have

$$
\phi_{ \pm}\left(\mathcal{L}_{\mp}^{(i)}\right)=B_{\mp}^{(i)}
$$

Proof Substituting $B_{+}^{(i)}$ into equations (18)-(20) and $B_{-}^{(i)}$ into equations (18)-(20) with $\varepsilon$ replaced by $-\varepsilon$, and using (21)-(24) the first claim follows immediately. The second claim is the result of a straightforward (symbolic) computation using Maple. $\square$

The map $\phi_{+}$blows down the lines $\mathcal{L}_{-}^{(i)}$ to the points $B_{-}^{(i)}$ and blows up the points $B_{+}^{(i)}$ to the lines $\mathcal{L}_{+}^{(i)}$.

Theorem 3.3 (i) Suppose that $n \gamma_{i} \neq \gamma_{j}+\gamma_{k}$, for $0 \leqslant n<N$. Then we have

$$
\phi_{+}^{n}\left(B_{-}^{(i)}\right)=\left[-\frac{b_{i}}{\varepsilon d_{i j} d_{k i}}, \frac{a_{i}}{\varepsilon d_{i j} d_{k i}},-2 n \gamma_{i}+\gamma_{j}+\gamma_{k}\right], \quad 0 \leqslant n \leqslant N,
$$

where $(i, j, k)$ is a cyclic permutation of $(1,2,3)$. In particular, we have

$$
\phi_{+}^{n_{i}-1}\left(B_{-}^{(i)}\right)=B_{+}^{(i)}
$$

if and only if

$$
\left(n_{i}-1\right) \gamma_{i}=\gamma_{j}+\gamma_{k}
$$

for a positive integer $n_{i} \in \mathbb{N}$.

(ii) The only orbit data with exactly three singular orbits that can be realized is $\left(\sigma_{1}, \sigma_{2}, \sigma_{3}\right)=(1,2,3)$ and (up to permutation)

$$
\begin{aligned}
& \left(n_{1}, n_{2}, n_{3}\right)=(3,3,3) \text { if and only if }\left(\gamma_{1}, \gamma_{2}, \gamma_{3}\right)=\lambda(1,1,1), \\
& \left(n_{1}, n_{2}, n_{3}\right)=(4,4,2) \text { if and only if }\left(\gamma_{1}, \gamma_{2}, \gamma_{3}\right)=\lambda(1,1,2), \\
& \left(n_{1}, n_{2}, n_{3}\right)=(6,3,2) \text { if and only if }\left(\gamma_{1}, \gamma_{2}, \gamma_{3}\right)=\lambda(1,2,3),
\end{aligned}
$$

for $\lambda \in \mathbb{R} \backslash\{0\}$.

(iii) The only orbit data with exactly two singular orbits that can be realized is $\left(\sigma_{1}, \sigma_{2}\right)=(1,2)$ and

$$
\begin{aligned}
\left(n_{1}, n_{2}\right) \in N_{2}=\mathbb{N}^{2} \backslash\{(3,3) & ,(2,4),(4,2),(4,4), \\
& (2,3),(3,2),(2,6),(6,2),(3,6),(6,3)\}
\end{aligned}
$$

if and only if

$$
\left(\gamma_{1}, \gamma_{2}, \gamma_{3}\right)=\lambda\left(n_{2}, n_{1}, n_{1} n_{2}-n_{1}-n_{2}\right),
$$

for $\lambda \in \mathbb{R} \backslash\{0\}$. 
(iv) The only orbit data with exactly one singular orbit that can be realized is $\sigma_{1}=1$ and $n_{1} \in \mathbb{N}$ arbitrary.

Proof (i) We show (25) by induction on $n$. For $n=0$ the claim is true by Proposition 3.2. In the induction step (from $n<N$ to $n+1$ ) with (18)-(20) and (21)-(24) we find that

$$
\begin{aligned}
x^{\prime} & =-\frac{2\left(-n \gamma_{i}+\gamma_{j}+\gamma_{k}\right) b_{i}}{\varepsilon d_{i j} d_{k i}}, \\
y^{\prime} & =\frac{2\left(-n \gamma_{i}+\gamma_{j}+\gamma_{k}\right) a_{i}}{\varepsilon d_{i j} d_{k i}}, \\
z^{\prime} & =2\left(-n \gamma_{i}+\gamma_{j}+\gamma_{k}\right)\left(-2(n+1) \gamma_{i}+\gamma_{j}+\gamma_{k}\right) .
\end{aligned}
$$

Since $n \gamma_{i} \neq \gamma_{j}+\gamma_{k}$, we find that

$$
\phi_{+}\left(\phi_{+}^{n}\left(B_{-}^{(i)}\right)\right)=\left[-\frac{b_{i}}{\varepsilon d_{i j} d_{k i}}, \frac{a_{i}}{\varepsilon d_{i j} d_{k i}},-2(n+1) \gamma_{i}+\gamma_{j}+\gamma_{k}\right] .
$$

This proves the claim.

(ii) From conditions (26), for $i=1,2,3$, we obtain the linear system

$$
\left(\begin{array}{ccc}
n_{1}-1 & -1 & -1 \\
-1 & n_{2}-1 & -1 \\
-1 & -1 & n_{3}-1
\end{array}\right)\left(\begin{array}{l}
\gamma_{1} \\
\gamma_{2} \\
\gamma_{3}
\end{array}\right)=\left(\begin{array}{l}
0 \\
0 \\
0
\end{array}\right)
$$

This system has nontrivial solutions if and only if

$$
\frac{1}{n_{1}}+\frac{1}{n_{2}}+\frac{1}{n_{3}}=1
$$

Equation (27) famously appears in the classification of tessellations of the Euclidean plane by congruent triangles. Indeed, the triangles of such a tessellation all have interior angles $\pi / n_{1}, \pi / n_{2}, \pi / n_{3}$ satisfying (27), so that the following triples $\left(n_{1}, n_{2}, n_{3}\right)$ are admissible:

$$
(3,3,3), \quad(4,4,2), \quad(6,3,2) \text {. }
$$

(iii) From conditions (26), for $i=1,2$, we obtain the linear system

$$
\left(\begin{array}{ccr}
n_{1}-1 & -1 & -1 \\
-1 & n_{2}-1 & -1
\end{array}\right)\left(\begin{array}{l}
\gamma_{1} \\
\gamma_{2} \\
\gamma_{3}
\end{array}\right)=\left(\begin{array}{l}
0 \\
0
\end{array}\right)
$$

Note that we have to exclude those values $\left(n_{1}, n_{2}\right) \in \mathbb{N}^{2}$ for which the solutions $\left(\gamma_{1}, \gamma_{2}, \gamma_{3}\right)$ correspond to orbit data with three singular orbits. This yields the proof. 
(iv) From conditions (26), for $i=1$, we obtain the linear equation

$$
\left(n_{1}-1-1-1\right)\left(\begin{array}{l}
\gamma_{1} \\
\gamma_{2} \\
\gamma_{3}
\end{array}\right)=0
$$

This yields the proof.

We arrive at the following classification result (compare Theorem 1.1):

Theorem 3.4 The sequence of degrees $d(m)$ of iterates $\phi_{+}^{m}$ grows exponentially, so that the map $\phi_{+}$is non-integrable, except for the following cases:

(i) If $\left(\gamma_{1}, \gamma_{2}, \gamma_{3}\right)=(1,1,1),(1,1,2),(1,2,3)$, the sequence $d(m)$ of degrees grows quadratically. The map $\phi_{+}$admits an invariant pencil of elliptic curves. The degree of a generic curve of the pencil is 3, 4, 6, respectively.

(ii) If $\left(\gamma_{1}, \gamma_{2}, \gamma_{3}\right)=(1,1,0)$ or $\left(\gamma_{1}, \gamma_{2}, \gamma_{3}\right)=(\alpha, 1,-1), \alpha \in \mathbb{R} \backslash \mathbb{Z} \cup\{0\}$, the sequence of degrees $d(m)$ grows linearly. The map $\phi_{+}$admits an invariant pencil of rational curves.

(iii) If $\left(\gamma_{1}, \gamma_{2}, \gamma_{3}\right)=(n, 1,-1), n \in \mathbb{N}$, the sequence of degrees $d(m)$ is bounded.

Here, $\left(\gamma_{1}, \gamma_{2}, \gamma_{3}\right)$ are fixed up to permutation and multiplication by $\lambda \in \mathbb{R} \backslash\{0\}$.

Proof We distinguish the number of singular orbits $s=0,1,2,3$ of the map $\phi_{+}$. $\boldsymbol{s}=\mathbf{3}$. If $\left(\gamma_{1}, \gamma_{2}, \gamma_{3}\right)=(1,1,1),(1,1,2),(1,2,3)$, the generating functions of the sequences of degrees are given by (39) and (47), respectively. The sequences $d(m)$ grow quadratically. The invariant pencils of elliptic curves are given by (29), (34) and (40), respectively. By Theorem 3.3 these are the only cases with three singular orbits. $s=2$. If $\left(\gamma_{1}, \gamma_{2}, \gamma_{3}\right)=(1,1,0)$, the sequence of degrees is given by (51). The sequence $d(m)$ grows linearly. The invariant pencil of rational curves is given by (48). If $\left(\gamma_{1}, \gamma_{2}, \gamma_{3}\right)=(n, 1,-1), n \in \mathbb{N}$, the generating function of the sequence of degrees is given by (54). The sequence $d(m)$ is bounded. By Theorem 3.3 all other cases with two singular orbits have orbit data $\left(\sigma_{1}, \sigma_{2}\right)=(1,2),\left(n_{1}, n_{2}\right)=(2+i, 2+j)$ with $i+j>2$. With [2, Theorem 3.3] and [1, Theorem 5.1] it follows that in those cases $\lambda_{1}>1$, i.e., the sequence $d(m)$ grows exponentially.

$\boldsymbol{s}=\mathbf{1}$. If $\left(\gamma_{1}, \gamma_{2}, \gamma_{3}\right)=(\alpha, 1,-1), \alpha \in \mathbb{R} \backslash \mathbb{Z} \cup\{0\}$, by Theorem 3.3 and (28) we have the orbit data $\sigma_{1}=1, n_{1}=1$. With Theorem 2.3 we find that the sequence $d(m)$ grows linearly. The claim about the existence of an invariant pencil of rational curves follows from Theorem 2.2. With (28) we find that all other cases with one singular orbit have orbit data $\sigma_{1}=1, n_{1}>1$. With [2, Theorem 3.3] and [1, Theorem 5.1] it follows that in those cases $\lambda_{1}>1$, i.e., the sequence $d(m)$ grows exponentially. $\boldsymbol{s}=\mathbf{0}$. We have $\lambda_{1}=2$. The sequence $d(m)$ grows exponentially.

\section{The case $\left(\gamma_{1}, \gamma_{2}, \gamma_{3}\right)=(1,1,1)$}

By Theorem 3.3 this case corresponds to the orbit data $\left(n_{1}, n_{2}, n_{3}\right)=(3,3,3)$, $\left(\sigma_{1}, \sigma_{2}, \sigma_{3}\right)=(1,2,3)$. In this case, we consider the Kahan map $\phi_{+}: \mathbb{C}^{2} \rightarrow \mathbb{C}^{2}$ 
corresponding to a quadratic vector field of the form

$$
\dot{\mathbf{x}}=J \nabla H(\mathbf{x}), \quad H(\mathbf{x})=\ell_{1}(\mathbf{x}) \ell_{2}(\mathbf{x}) \ell_{3}(\mathbf{x}) .
$$

The Kahan map $\phi_{+}: \mathbb{C}^{2} \rightarrow \mathbb{C}^{2}$ admits an integral of motion (see $[4,18]$ ):

$$
\widetilde{H}(\mathbf{x})=\frac{H(\mathbf{x})}{Q(\mathbf{x})},
$$

where

$$
Q(\mathbf{x})=1+4 \varepsilon^{2}\left(\left(d_{1} d_{3}-d_{2}^{2}\right) x^{2}+\left(d_{1} d_{4}-d_{2} d_{3}\right) x y+\left(d_{2} d_{4}-d_{3}^{2}\right) y^{2}\right),
$$

with $d_{1}=3 a_{1} a_{2} a_{3}, d_{2}=a_{1} a_{2} b_{3}+a_{1} a_{3} b_{2}+a_{2} a_{3} b_{1}, d_{3}=a_{3} b_{1} b_{2}+a_{2} b_{1} b_{3}+a_{1} b_{2} b_{3}$, $d_{4}=3 b_{1} b_{2} b_{3}$.

The geometry of the Kahan discretization was studied in [18]. The phase space of $\phi_{+}: \mathbb{C}^{2} \rightarrow \mathbb{C}^{2}$ is foliated by the one-parameter family (pencil) of invariant curves

$$
\mathcal{E}_{\lambda}=\left\{(x, y) \in \mathbb{C}^{2}: H(x, y)-\lambda Q(x, y)=0\right\} .
$$

We consider $\mathbb{C}^{2}$ as an affine part of $\mathbb{C P}^{2}$ consisting of the points $[x, y, z] \in \mathbb{C P}^{2}$ with $z \neq 0$. We define the projective curves $\overline{\mathcal{E}}_{\lambda}$ as projective completion of $\mathcal{E}_{\lambda}$ :

$$
\overline{\mathcal{E}}_{\lambda}=\left\{[x, y, z] \in \mathbb{C P}^{2}: H(x, y)-\lambda z \bar{Q}(x, y, z)=0\right\},
$$

where we set

$$
\bar{Q}(x, y, z)=z^{2} Q(x / z, y / z) .
$$

(We have $\bar{H}(x, y, z)=z^{3} H(x / z, y / z)=H(x, y)$ since $H(x, y)$ is homogeneous of degree three.) The pencil has deg $=3$ and contains two reducible curves

$$
\overline{\mathcal{E}}_{0}=\left\{[x, y, z] \in \mathbb{C P}^{2}: H(x, y)=0\right\}
$$

consisting of the lines $\left\{\ell_{i}(x, y)=0\right\}, i=1,2,3$, and

$$
\overline{\mathcal{E}}_{\infty}=\left\{[x, y, z] \in \mathbb{C P}^{2}: z \bar{Q}(x, y, z)=0\right\}
$$

consisting of the conic $\{\bar{Q}(x, y, z)=0\}$ and the line at infinity $\{z=0\}$. All curves $\overline{\mathcal{E}}_{\lambda}$ pass through the set of base points which is defined as $\overline{\mathcal{E}}_{0} \cap \overline{\mathcal{E}}_{\infty}$. According to the Bézout theorem, there are nine base points, counted with multiplicities.

Proposition 4.1 The nine base points are given by:

- two finite base points of multiplicity 1 on each of the lines $\ell_{i}=0, i=1,2,3$ :

$$
B_{ \pm}^{(i)}=\left( \pm \frac{b_{i}}{2 \varepsilon d_{i j} d_{k i}}, \mp \frac{a_{i}}{2 \varepsilon d_{i j} d_{k i}}\right)
$$




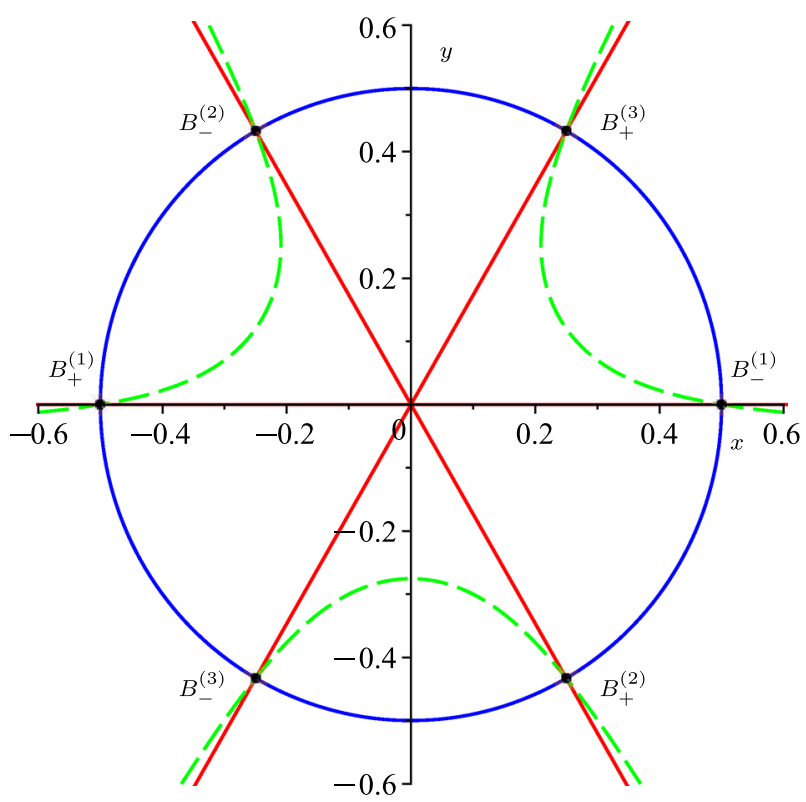

Fig. 1 The curves $\overline{\mathcal{E}}_{0}, \overline{\mathcal{E}}_{\infty}, \overline{\mathcal{E}}_{0.01}$ in resp. red, blue and green for $H(x, y)=H_{1}(x, y), \varepsilon=1$ (Color figure online)

- one base point of multiplicity 1 at infinity on each of the lines $\ell_{i}=0, i=1,2,3$ :

$$
F^{(i)}=\left[b_{i},-a_{i}, 0\right] .
$$

The singular orbits of the map are as follows:

$$
\begin{aligned}
& \mathcal{L}_{-}^{(1)} \longrightarrow B_{-}^{(1)} \longrightarrow F^{(1)} \longrightarrow B_{+}^{(1)} \longrightarrow \mathcal{L}_{+}^{(1)}, \\
& \mathcal{L}_{-}^{(2)} \longrightarrow B_{-}^{(2)} \longrightarrow F^{(2)} \longrightarrow B_{+}^{(2)} \longrightarrow \mathcal{L}_{+}^{(2)}, \\
& \mathcal{L}_{-}^{(3)} \longrightarrow B_{-}^{(3)} \longrightarrow F^{(3)} \longrightarrow B_{+}^{(3)} \longrightarrow \mathcal{L}_{+}^{(3)},
\end{aligned}
$$

where $\mathcal{L}_{\mp}^{(i)}$ denotes the line through the points $B_{ \pm}^{(j)}, B_{ \pm}^{(k)}$.

Proof The singular orbits (32) are a consequence of Proposition 3.2 and Theorem 3.3. It can be verified by straightforward computations that the points (30)-(31) are base points of the pencil of invariant curves $\overline{\mathcal{E}}_{\lambda}$.

\subsection{Lifting the map to a surface automorphism}

We blow up the plane $\mathbb{C} \mathbb{P}^{2}$ at the nine base points $B_{-}^{(i)}, F^{(i)}, B_{+}^{(i)}, i=1,2,3$, and denote the corresponding exceptional divisors by $E_{i, 0}, E_{i, 1}, E_{i, 2}, i=1,2,3$. The resulting blow-up surface is denoted by $X$. On this surface $\phi_{+}$is lifted to an automorphism $\widetilde{\phi}_{+}$ 
acting on the exceptional divisors according to the scheme [compare with (32)]

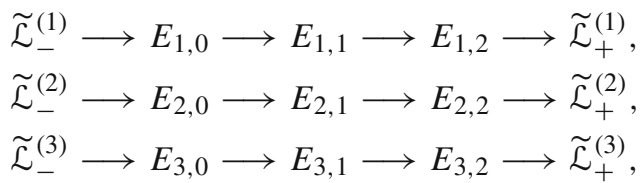

where $\widetilde{\mathcal{L}}_{ \pm}^{(i)}$ denotes the proper transform of the line $\mathcal{L}_{ \pm}^{(i)}$.

We compute the induced pullback map on the Picard group $\widetilde{\phi}_{+}^{*}: \operatorname{Pic}(X) \rightarrow \operatorname{Pic}(X)$. Let $\mathcal{H} \in \operatorname{Pic}(X)$ be the pullback of the class of a generic line in $\mathbb{C P}^{2}$. Let $\mathcal{E}_{i, n} \in \operatorname{Pic}(X)$, for $i \leqslant 3$ and $0 \leqslant n \leqslant 2$, be the class of $E_{i, n}$. Then the Picard group is

$$
\operatorname{Pic}(X)=\mathbb{Z} \mathcal{H} \bigoplus_{i=1}^{3} \bigoplus_{n=0}^{2} \mathbb{Z} \varepsilon_{i, n} .
$$

The rank of the Picard group is 10 . The induced pullback $\widetilde{\phi}_{+}^{*}: \operatorname{Pic}(X) \rightarrow \operatorname{Pic}(X)$ is determined by (3).

With Theorem 2.3 we arrive at the system of recurrence relations for the degree $d(m)$ :

$$
\left\{\begin{array}{l}
d(m+1)=2 d(m)-\mu_{1}(m)-\mu_{2}(m)-\mu_{3}(m), \\
\mu_{1}(m+3)=d(m)-\mu_{2}(m)-\mu_{3}(m), \\
\mu_{2}(m+3)=d(m)-\mu_{1}(m)-\mu_{3}(m), \\
\mu_{3}(m+3)=d(m)-\mu_{1}(m)-\mu_{2}(m),
\end{array}\right.
$$

with initial conditions $d(0)=1, \mu_{i}(m)=0$, for $m=0, \ldots, 2, i=1,2,3$. The generating functions of the solution to this system of recurrence relations are given by

$$
\begin{aligned}
d(z) & =-\frac{2 z^{3}+1}{(z+1)(z-1)^{3}}, \\
\mu_{i}(z) & =-\frac{z^{3}}{(z+1)(z-1)^{3}}, \quad i=1,2,3 .
\end{aligned}
$$

The sequence $d(m)$ grows quadratically.

\section{The case $\left(\gamma_{1}, \gamma_{2}, \gamma_{3}\right)=(1,1,2)$}

By Theorem 3.3 this case corresponds to the orbit data $\left(n_{1}, n_{2}, n_{3}\right)=(4,4,2)$, $\left(\sigma_{1}, \sigma_{2}, \sigma_{3}\right)=(1,2,3)$. In this case, we consider the Kahan map $\phi_{+}: \mathbb{C}^{2} \rightarrow \mathbb{C}^{2}$ corresponding to a quadratic vector field of the form

$$
\dot{\mathbf{x}}=\frac{1}{\ell_{3}(\mathbf{x})} J \nabla H(\mathbf{x}), \quad H(\mathbf{x})=\ell_{1}(\mathbf{x}) \ell_{2}(\mathbf{x}) \ell_{3}^{2}(\mathbf{x}) .
$$


The Kahan map $\phi_{+}: \mathbb{C}^{2} \rightarrow \mathbb{C}^{2}$ admits an integral of motion (see $[6,20]$ ):

$$
\widetilde{H}(\mathbf{x})=\frac{H(\mathbf{x})}{P_{1}(\mathbf{x}) P_{2}(\mathbf{x}) Q(\mathbf{x})},
$$

where

$$
\begin{aligned}
& P_{1}(\mathbf{x})=1+\varepsilon\left(d_{23} \ell_{1}(\mathbf{x})-d_{31} \ell_{2}(\mathbf{x})\right), \\
& P_{2}(\mathbf{x})=1-\varepsilon\left(d_{23} \ell_{1}(\mathbf{x})-d_{31} \ell_{2}(\mathbf{x})\right), \\
& Q(\mathbf{x})=1-\varepsilon^{2}\left(9 d_{12}^{2} \ell_{3}^{2}(\mathbf{x})-4 d_{23} d_{31} \ell_{1}(\mathbf{x}) \ell_{2}(\mathbf{x})\right) .
\end{aligned}
$$

The phase space of $\phi_{+}: \mathbb{C}^{2} \rightarrow \mathbb{C}^{2}$ is foliated by the one-parameter family (pencil) of invariant curves

$$
\mathcal{E}_{\lambda}=\left\{(x, y) \in \mathbb{C}^{2}: H(x, y)-\lambda P_{1}(x, y) P_{2}(x, y) Q(x, y)=0\right\} .
$$

We define the projective curves $\overline{\mathcal{E}}_{\lambda}$ as projective completion of $\mathcal{E}_{\lambda}$ :

$$
\overline{\mathcal{E}}_{\lambda}=\left\{[x, y, z] \in \mathbb{C P}^{2}: H(x, y)-\lambda \bar{P}_{1}(x, y, z) \bar{P}_{2}(x, y, z) \bar{Q}(x, y, z)=0\right\},
$$

where we set

$$
\bar{P}_{i}(x, y, z)=z P_{i}(x / z, y / z), \quad i=1,2, \quad \bar{Q}(x, y, z)=z^{2} Q(x / z, y / z) .
$$

The pencil has deg $=4$ and contains two reducible curves

$$
\overline{\mathcal{E}}_{0}=\left\{[x, y, z] \in \mathbb{C P}^{2}: H(x, y)=0\right\}
$$

consisting of the lines $\left\{\ell_{i}(x, y)=0\right\}, i=1,2,3$, with multiplicities $1,1,2$, and

$$
\overline{\mathcal{E}}_{\infty}=\left\{[x, y, z] \in \mathbb{C P}^{2}: \bar{P}_{1}(x, y, z) \bar{P}_{2}(x, y, z) \bar{Q}(x, y, z)=0\right\}
$$

consisting of the two lines $\left\{\bar{P}_{i}(x, y, z)=0\right\}, i=1,2$, and the conic $\{\bar{Q}(x, y, z)=0\}$. All curves $\overline{\mathcal{E}}_{\lambda}$ pass through the set of base points which is defined as $\overline{\mathcal{E}}_{0} \cap \overline{\mathcal{E}}_{\infty}$.

Proposition 5.1 The ten (distinct) base points are given by:

- four base points of multiplicity 1 on each of the lines $\ell_{i}=0, i=1,2$ :

$$
\begin{aligned}
& B_{ \pm}^{(i)}=\left( \pm \frac{b_{i}}{3 \varepsilon d_{i j} d_{k i}}, \mp \frac{a_{i}}{3 \varepsilon d_{i j} d_{k i}}\right) \\
& C_{ \pm}^{(i)}=\left( \pm \frac{b_{i}}{\varepsilon d_{i j} d_{k i}}, \mp \frac{a_{i}}{\varepsilon d_{i j} d_{k i}}\right)
\end{aligned}
$$


- two base points of multiplicity 2 on the line $\ell_{3}=0$ :

$$
B_{ \pm}^{(3)}=\left( \pm \frac{b_{3}}{2 \varepsilon d_{23} d_{31}}, \mp \frac{a_{3}}{2 \varepsilon d_{23} d_{31}}\right) \text {. }
$$

The singular orbits of the map are as follows:

$$
\begin{aligned}
& \mathcal{L}_{-}^{(1)} \longrightarrow B_{-}^{(1)} \longrightarrow C_{-}^{(1)} \longrightarrow C_{+}^{(1)} \longrightarrow B_{+}^{(1)} \longrightarrow \mathcal{L}_{+}^{(1)}, \\
& \mathcal{L}_{-}^{(2)} \longrightarrow B_{-}^{(2)} \longrightarrow C_{-}^{(2)} \longrightarrow C_{+}^{(2)} \longrightarrow B_{+}^{(2)} \longrightarrow \mathcal{L}_{+}^{(2)}, \\
& \mathcal{L}_{-}^{(3)} \longrightarrow B_{-}^{(3)} \longrightarrow B_{+}^{(3)} \longrightarrow \mathcal{L}_{+}^{(3)},
\end{aligned}
$$

where $\mathcal{L}_{\mp}^{(i)}$ denotes the line through the points $B_{ \pm}^{(j)}, B_{ \pm}^{(k)}$.

Proof The singular orbits (38) are a consequence of Proposition 3.2 and Theorem 3.3. It can be verified by straightforward computations that the points (35)-(37) are base points of the pencil of invariant curves $\overline{\mathcal{E}}_{\lambda}$.

According to the Bézout theorem, there are 16 base points, counted with multiplicities. This number is obtained by

$$
\sum_{P \in \overline{\mathcal{E}}_{0} \cap \overline{\mathcal{E}}_{\infty}}(\operatorname{mult}(P))^{2}=8 \cdot 1+2 \cdot 4
$$

where mult $(P)$ denotes the multiplicity of the base point $P$.

\subsection{Lifting the map to a surface automorphism}

We blow up the plane $\mathbb{C P}^{2}$ at the ten base points $B_{-}^{(i)}, B_{+}^{(i)}, i=1,2,3$, and $C_{-}^{(i)}, C_{+}^{(i)}$, $i=1,2$, and denote the corresponding exceptional divisors by $E_{i, 0}, \ldots, E_{i, n_{i}-1}$, $i=1,2,3$. The resulting blow-up surface is denoted by $X$. On this surface $\phi_{+}$is lifted to an automorphism $\widetilde{\phi}_{+}$acting on the exceptional divisors according to the scheme [compare with (38)]

$$
\begin{aligned}
& \tilde{\mathcal{L}}_{-}^{(1)} \longrightarrow E_{1,0} \longrightarrow E_{1,1} \longrightarrow E_{1,2} \longrightarrow E_{1,3} \longrightarrow \tilde{\mathcal{L}}_{+}^{(1)}, \\
& \tilde{\mathcal{L}}_{-}^{(2)} \longrightarrow E_{2,0} \longrightarrow E_{2,1} \longrightarrow E_{2,2} \longrightarrow E_{2,3} \longrightarrow \tilde{\mathcal{L}}_{+}^{(2)}, \\
& \tilde{\mathcal{L}}_{-}^{(3)} \longrightarrow E_{3,0} \longrightarrow E_{3,1} \longrightarrow \tilde{\mathcal{L}}_{+}^{(3)},
\end{aligned}
$$

where $\widetilde{\mathcal{L}}_{ \pm}^{(i)}$ denotes the proper transform of the line $\mathcal{L}_{ \pm}^{(i)}$.

We compute the induced pullback map on the Picard group $\widetilde{\phi}_{+}^{*}: \operatorname{Pic}(X) \rightarrow \operatorname{Pic}(X)$. Let $\mathcal{H} \in \operatorname{Pic}(X)$ be the pullback of the class of a generic line in $\mathbb{C P}^{2}$. Let $\mathcal{E}_{i, n} \in \operatorname{Pic}(X)$, for $i \leqslant 3$ and $0 \leqslant n \leqslant n_{i}-1$, be the class of $E_{i, n}$. Then the Picard group is

$$
\operatorname{Pic}(X)=\mathbb{Z} \mathcal{H} \bigoplus_{i=1}^{3} \bigoplus_{n=0}^{n_{i}-1} \mathbb{Z} \mathcal{E}_{i, n} .
$$




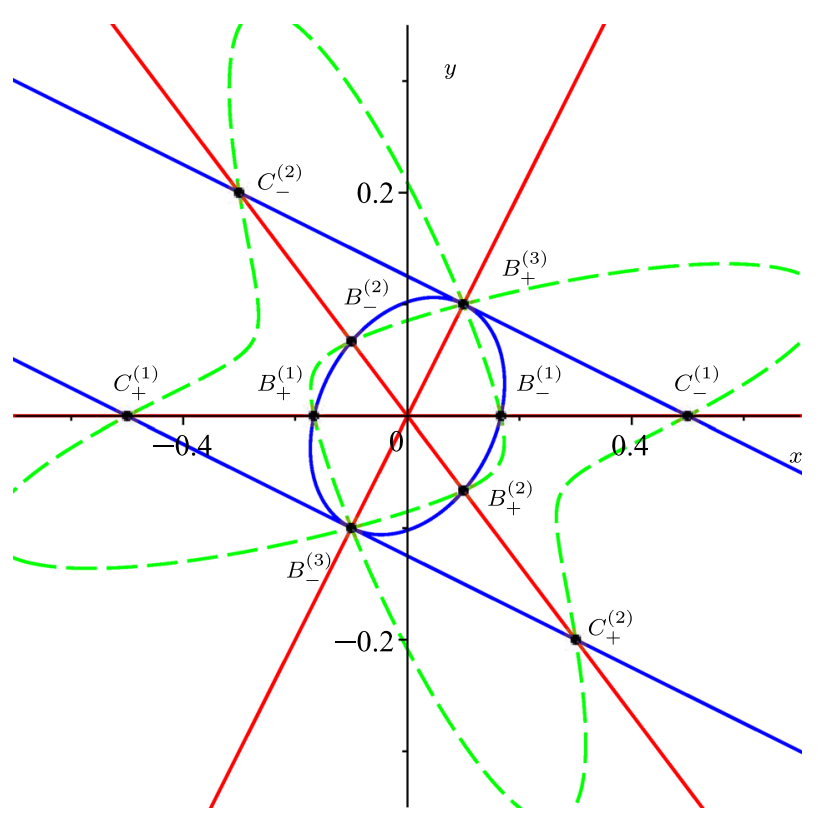

Fig. 2 The curves $\overline{\mathcal{E}}_{0}, \overline{\mathcal{E}}_{\infty}, \overline{\mathcal{E}}_{0.001}$ in resp. red, blue and green for $H(x, y)=H_{2}(x, y), \varepsilon=1$ (Color figure online)

The rank of the Picard group is 11 . The induced pullback $\widetilde{\phi}_{+}^{*}: \operatorname{Pic}(X) \rightarrow \operatorname{Pic}(X)$ is determined by (3).

With Theorem 2.3 we arrive at the system of recurrence relations for the degree $d(m)$ :

$$
\left\{\begin{array}{l}
d(m+1)=2 d(m)-\mu_{1}(m)-\mu_{2}(m)-\mu_{3}(m), \\
\mu_{1}(m+4)=d(m)-\mu_{2}(m)-\mu_{3}(m), \\
\mu_{2}(m+4)=d(m)-\mu_{1}(m)-\mu_{3}(m), \\
\mu_{3}(m+2)=d(m)-\mu_{1}(m)-\mu_{2}(m),
\end{array}\right.
$$

with initial conditions $d(0)=1, \mu_{i}(m)=0$, for $n=0, \ldots, 3, i=1,2$, and $\mu_{3}(m)=0$, for $m=0,1$. The generating functions of the solution to this system of recurrence relations are given by:

$$
\begin{aligned}
d(z) & =-\frac{2 z^{4}+z^{2}+1}{\left(z^{2}+z+1\right)(z-1)^{3}}, \\
\mu_{i}(z) & =-\frac{z^{4}}{\left(z^{2}+z+1\right)(z-1)^{3}}, \quad i=1,2, \\
\mu_{3}(z) & =-\frac{z^{2}\left(z^{2}+1\right)}{\left(z^{2}+z+1\right)(z-1)^{3}} .
\end{aligned}
$$

The sequence $d(m)$ grows quadratically. 


\section{The case $\left(\gamma_{1}, \gamma_{2}, \gamma_{3}\right)=(1,2,3)$}

By Theorem 3.3 this case corresponds to the orbit data $\left(n_{1}, n_{2}, n_{3}\right)=(6,3,2)$, $\left(\sigma_{1}, \sigma_{2}, \sigma_{3}\right)=(1,2,3)$. In this case, we consider the Kahan map $\phi_{+}: \mathbb{C}^{2} \rightarrow \mathbb{C}^{2}$ corresponding to a quadratic vector field of the form

$$
\dot{\mathbf{x}}=\frac{1}{\ell_{2}(\mathbf{x}) \ell_{3}^{2}(\mathbf{x})} J \nabla H(\mathbf{x}), \quad H(\mathbf{x})=\ell_{1}(\mathbf{x}) \ell_{2}^{2}(\mathbf{x}) \ell_{3}^{3}(\mathbf{x}) .
$$

The Kahan map $\phi_{+}: \mathbb{C}^{2} \rightarrow \mathbb{C}^{2}$ admits an integral of motion (see $\left.[6,20]\right)$ :

$$
\widetilde{H}(\mathbf{x})=\frac{H(\mathbf{x})}{P_{1}(\mathbf{x}) P_{2}(\mathbf{x}) P_{3}(\mathbf{x}) P_{4}(\mathbf{x}) Q(\mathbf{x})},
$$

where

$$
\begin{aligned}
& P_{1}(\mathbf{x})=1+3 \varepsilon d_{31} \ell_{2}(\mathbf{x}), \\
& P_{2}(\mathbf{x})=1-3 \varepsilon d_{31} \ell_{2}(\mathbf{x}), \\
& P_{3}(\mathbf{x})=1+\varepsilon\left(3 d_{23} \ell_{1}(\mathbf{x})-d_{12} \ell_{3}(\mathbf{x})\right), \\
& P_{4}(\mathbf{x})=1-\varepsilon\left(3 d_{23} \ell_{1}(\mathbf{x})-d_{12} \ell_{3}(\mathbf{x})\right), \\
& Q(\mathbf{x})=1-\varepsilon^{2}\left(9 d_{31}^{2} \ell_{2}^{2}(\mathbf{x})+16 d_{12}^{2} \ell_{3}^{2}(\mathbf{x})\right) .
\end{aligned}
$$

The phase space of $\phi_{+}: \mathbb{C}^{2} \rightarrow \mathbb{C}^{2}$ is foliated by the one-parameter family (pencil) of invariant curves

$$
\mathcal{E}_{\lambda}=\left\{(x, y) \in \mathbb{C}^{2}: H(x, y)-\lambda Q(x, y) \prod_{i=1}^{4} P_{i}(x, y)=0\right\} .
$$

We define the projective curves $\overline{\mathcal{E}}_{\lambda}$ as projective completion of $\mathcal{E}_{\lambda}$ :

$$
\overline{\mathcal{E}}_{\lambda}=\left\{[x, y, z] \in \mathbb{C P}^{2}: H(x, y)-\lambda \bar{Q}(x, y, z) \prod_{i=1}^{4} \bar{P}_{i}(x, y, z)=0\right\},
$$

where we set

$$
\bar{P}_{i}(x, y, z)=z P_{i}(x / z, y / z), \quad i=1, \ldots, 4, \quad \bar{Q}(x, y, z)=z^{2} Q(x / z, y / z) .
$$

The pencil has deg $=6$ and contains two reducible curves

$$
\overline{\mathcal{E}}_{0}=\left\{[x, y, z] \in \mathbb{C P}^{2}: H(x, y)=0\right\}
$$


consisting of the lines $\left\{\ell_{i}(x, y)=0\right\}, i=1,2,3$, with multiplicities $1,2,3$, and

$$
\overline{\mathcal{E}}_{\infty}=\left\{[x, y, z] \in \mathbb{C P}^{2}: \bar{Q}(x, y, z) \prod_{i=1}^{4} \bar{P}_{i}(x, y, z)=0\right\}
$$

consisting of the four lines $\left\{\bar{P}_{i}(x, y, z)=0\right\}, i=1, \ldots, 4$, and the conic $\{\bar{Q}(x, y, z)=0\}$. All curves $\overline{\mathcal{E}}_{\lambda}$ pass through the set of base points which is defined as $\overline{\mathcal{E}}_{0} \cap \overline{\mathcal{E}}_{\infty}$.

Proposition 6.1 The eleven (distinct) base points are given by:

- six finite base points of multiplicity 1 on the line $\ell_{1}=0$ :

$$
\begin{aligned}
& B_{ \pm}^{(1)}=\left( \pm \frac{b_{1}}{5 \varepsilon d_{12} d_{31}}, \mp \frac{a_{1}}{5 \varepsilon d_{12} d_{31}}\right) \\
& C_{ \pm}^{(1)}=\left( \pm \frac{b_{1}}{3 \varepsilon d_{12} d_{31}}, \mp \frac{a_{1}}{3 \varepsilon d_{12} d_{31}}\right), \quad D_{ \pm}^{(1)}=\left( \pm \frac{b_{1}}{\varepsilon d_{12} d_{31}}, \mp \frac{a_{1}}{\varepsilon d_{12} d_{31}}\right)
\end{aligned}
$$

- two finite base points of multiplicity 2 on the line $\ell_{2}=0$ :

$$
B_{ \pm}^{(2)}=\left( \pm \frac{b_{2}}{4 \varepsilon d_{12} d_{23}}, \mp \frac{a_{2}}{4 \varepsilon d_{12} d_{23}}\right)
$$

- one base point of multiplicity 2 at infinity on the line $\ell_{2}=0$ :

$$
F^{(2)}=\left[b_{2},-a_{2}, 0\right],
$$

- two finite base points of multiplicity 3 on the line $\ell_{3}=0$ :

$$
B_{ \pm}^{(3)}=\left( \pm \frac{b_{3}}{3 \varepsilon d_{23} d_{31}}, \mp \frac{a_{3}}{3 \varepsilon d_{23} d_{31}}\right) \text {. }
$$

The singular orbits of the map are as follows:

$$
\begin{aligned}
& \mathcal{L}_{-}^{(1)} \longrightarrow B_{-}^{(1)} \longrightarrow C_{-}^{(1)} \longrightarrow D_{-}^{(1)} \longrightarrow D_{+}^{(1)} \longrightarrow C_{+}^{(1)} \longrightarrow B_{+}^{(1)} \longrightarrow \mathcal{L}_{+}^{(1)}, \\
& \mathcal{L}_{-}^{(2)} \longrightarrow B_{-}^{(2)} \longrightarrow F^{(2)} \longrightarrow B_{+}^{(2)} \longrightarrow \mathcal{L}_{+}^{(2)}, \\
& \mathcal{L}_{-}^{(3)} \longrightarrow B_{-}^{(3)} \longrightarrow B_{+}^{(3)} \longrightarrow \mathcal{L}_{+}^{(3)}
\end{aligned}
$$

where $\mathcal{L}_{\mp}^{(i)}$ denotes the line through the points $B_{ \pm}^{(j)}, B_{ \pm}^{(k)}$.

Proof The singular orbits (46) are a consequence of Proposition 3.2 and Theorem 3.3. It can be verified by straightforward computations that the points (41)-(45) are base points of the pencil of invariant curves $\overline{\mathcal{E}}_{\lambda}$. 


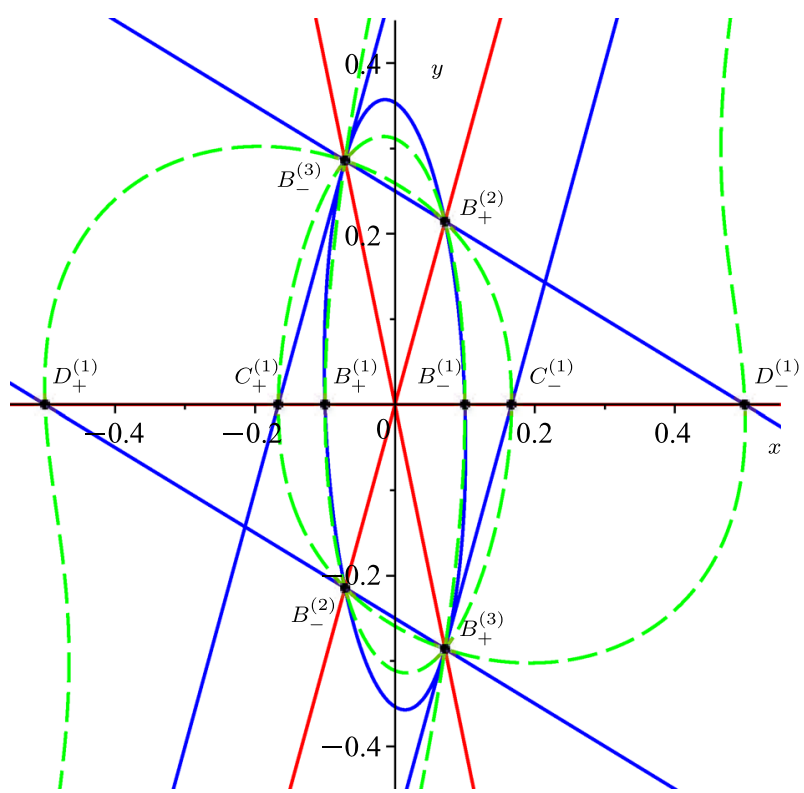

Fig. 3 The curves $\overline{\mathcal{E}}_{0}, \overline{\mathcal{E}}_{\infty}, \overline{\mathcal{E}}_{-0.002}$ in resp. red, blue and green for $H(x, y)=H_{3}(x, y), \varepsilon=1$ (Color figure online)

According to the Bézout theorem, there are 36 base points, counted with multiplicities. This number is obtained by

$$
\sum_{P \in \overline{\mathcal{E}}_{0} \cap \overline{\mathcal{E}}_{\infty}}(\operatorname{mult}(P))^{2}=6 \cdot 1+3 \cdot 4+2 \cdot 9 .
$$

\subsection{Lifting the map to a surface automorphism}

We blow up the plane $\mathbb{C P}^{2}$ at the eleven base points $B_{-}^{(i)}, B_{+}^{(i)}, i=1,2,3$, and $C_{-}^{(1)}, C_{+}^{(1)}, D_{-}^{(1)}, D_{+}^{(1)}$ and $F^{(2)}$ and denote the corresponding exceptional divisors by $E_{i, 0}, \ldots, E_{i, n_{i}-1}, i=1,2,3$. The resulting blow-up surface is denoted by $X$. On this surface $\phi_{+}$is lifted to an automorphism $\widetilde{\phi}_{+}$acting on the exceptional divisors according to the scheme [compare with (46)]

$$
\begin{aligned}
& \tilde{\mathcal{L}}_{-}^{(1)} \longrightarrow E_{1,0} \longrightarrow E_{1,1} \longrightarrow E_{1,2} \longrightarrow E_{1,3} \longrightarrow E_{1,4} \longrightarrow E_{1,5} \longrightarrow \tilde{\mathcal{L}}_{+}^{(1)}, \\
& \tilde{\mathcal{L}}_{-}^{(2)} \longrightarrow E_{2,0} \longrightarrow E_{2,1} \longrightarrow E_{2,2} \longrightarrow \tilde{\mathcal{L}}_{+}^{(2)}, \\
& \tilde{\mathcal{L}}_{-}^{(3)} \longrightarrow E_{3,0} \longrightarrow E_{3,1} \longrightarrow \tilde{\mathcal{L}}_{+}^{(3)},
\end{aligned}
$$

where $\widetilde{\mathcal{L}}_{ \pm}^{(i)}$ denotes the proper transform of the line $\mathcal{L}_{ \pm}^{(i)}$. 
We compute the induced pullback map on the Picard group $\widetilde{\phi}_{+}^{*}: \operatorname{Pic}(X) \rightarrow \operatorname{Pic}(X)$. Let $\mathcal{H} \in \operatorname{Pic}(X)$ be the pullback of the class of a generic line in $\mathbb{C P}^{2}$. Let $\mathcal{E}_{i, n} \in \operatorname{Pic}(X)$, for $i \leqslant 3$ and $0 \leqslant n \leqslant n_{i}-1$, be the class of $E_{i, n}$. Then the Picard group is

$$
\operatorname{Pic}(X)=\mathbb{Z} \mathcal{H} \bigoplus_{i=1}^{3} \bigoplus_{n=0}^{n_{i}-1} \mathbb{Z} \mathcal{E}_{i, n}
$$

The rank of the Picard group is 12 . The induced pullback $\widetilde{\phi}_{+}^{*}: \operatorname{Pic}(X) \rightarrow \operatorname{Pic}(X)$ is determined by (3).

With Theorem 2.3 we arrive at the system of recurrence relations for the degree $d(m)$ :

$$
\left\{\begin{array}{l}
d(m+1)=2 d(m)-\mu_{1}(m)-\mu_{2}(m)-\mu_{3}(m) \\
\mu_{1}(m+6)=d(m)-\mu_{2}(m)-\mu_{3}(m) \\
\mu_{2}(m+3)=d(m)-\mu_{1}(m)-\mu_{3}(m) \\
\mu_{3}(m+2)=d(m)-\mu_{1}(m)-\mu_{2}(m)
\end{array}\right.
$$

with initial conditions $d(0)=1, \mu_{1}(m)=0$, for $m=0, \ldots, 5, \mu_{2}(m)=0$, for $m=0,1,2$, and $\mu_{3}(m)=0$, for $m=0,1$. The generating functions of the solution to this system of recurrence relations are given by:

$$
\begin{aligned}
d(z) & =-\frac{2 z^{6}+z^{4}+z^{3}+z^{2}+1}{\left(z^{4}+z^{3}+z^{2}+z+1\right)(z-1)^{3}}, \\
\mu_{1}(z) & =-\frac{z^{6}}{\left(z^{4}+z^{3}+z^{2}+z+1\right)(z-1)^{3}}, \\
\mu_{2}(z) & =-\frac{z^{3}(z+1)\left(z^{2}-z+1\right)}{\left(z^{4}+z^{3}+z^{2}+z+1\right)(z-1)^{3}}, \\
\mu_{3}(z) & =-\frac{z^{2}\left(z^{2}+z+1\right)\left(z^{2}-z+1\right)}{\left(z^{4}+z^{3}+z^{2}+z+1\right)(z-1)^{3}} .
\end{aligned}
$$

The sequence $d(m)$ grows quadratically.

\section{The case $\left(\gamma_{1}, \gamma_{2}, \gamma_{3}\right)=(1,1,0)$}

By Theorem 3.3 this case corresponds to the orbit data $\left(n_{1}, n_{2}\right)=(2,2),\left(\sigma_{1}, \sigma_{2}\right)=$ $(1,2)$. In this case, we consider the Kahan map $\phi_{+}: \mathbb{C}^{2} \rightarrow \mathbb{C}^{2}$ corresponding to a quadratic vector field of the form

$$
\dot{\mathbf{x}}=\ell_{3}(\mathbf{x}) J \nabla H(\mathbf{x}), \quad H(\mathbf{x})=\ell_{1}(\mathbf{x}) \ell_{2}(\mathbf{x}) .
$$


For $\ell_{1}(\mathbf{x})=x+y, \ell_{2}(\mathbf{x})=x-y, \ell_{3}(\mathbf{x})=x$ the vector field reads

$$
\left\{\begin{array}{l}
\dot{x}=-2 x y \\
\dot{y}=-2 x^{2}
\end{array}\right.
$$

and the Kahan discretization (13) reads

$$
\left\{\begin{array}{l}
\tilde{x}-x=-2 \varepsilon(\tilde{x} y+x \tilde{y}), \\
\tilde{y}-y=-4 \varepsilon \tilde{x} x .
\end{array}\right.
$$

The Kahan map $\phi_{+}: \mathbb{C}^{2} \rightarrow \mathbb{C}^{2}$ admits an integral of motion (see $[5,14]$ ):

$$
\widetilde{H}(\mathbf{x})=\frac{\ell_{1}(\mathbf{x}) \ell_{2}(\mathbf{x})}{P_{1}(\mathbf{x}) P_{2}(\mathbf{x})},
$$

where

$$
\begin{aligned}
& P_{1}(\mathbf{x})=1+\varepsilon d_{12} \ell_{3}(\mathbf{x}), \\
& P_{2}(\mathbf{x})=1-\varepsilon d_{12} \ell_{3}(\mathbf{x}) .
\end{aligned}
$$

The geometry of the Kahan discretization was studied in [19]. The phase space of $\phi_{+}: \mathbb{C}^{2} \rightarrow \mathbb{C}^{2}$ is foliated by the one-parameter family (pencil) of invariant curves

$$
\mathcal{E}_{\lambda}=\left\{(x, y) \in \mathbb{C}^{2}: H(x, y)-\lambda P_{1}(x, y) P_{2}(x, y)=0\right\} .
$$

We define the projective curves $\overline{\mathcal{E}}_{\lambda}$ as projective completion of $\mathcal{E}_{\lambda}$ :

$$
\overline{\mathcal{E}}_{\lambda}=\left\{[x, y, z] \in \mathbb{C P}^{2}: H(x, y)-\lambda \bar{P}_{1}(x, y, z) \bar{P}_{2}(x, y, z)=0\right\},
$$

where we set

$$
\bar{P}_{i}(x, y, z)=z P_{i}(x / z, y / z), \quad i=1,2 .
$$

The pencil has deg $=2$ and contains two reducible curves

$$
\overline{\mathcal{E}}_{0}=\left\{[x, y, z] \in \mathbb{C P}^{2}: H(x, y)=0\right\}
$$

consisting of the lines $\left\{\ell_{i}(x, y)=0\right\}, i=1,2$, and

$$
\overline{\mathcal{E}}_{\infty}=\left\{[x, y, z] \in \mathbb{C P}^{2}: \bar{P}_{1}(x, y, z) \bar{P}_{2}(x, y, z)=0\right\}
$$

consisting of the two lines $\left\{\bar{P}_{i}(x, y, z)=0\right\}, i=1$, 2. All curves $\overline{\mathcal{E}}_{\lambda}$ pass through the set of base points which is defined as $\overline{\mathcal{E}}_{0} \cap \overline{\mathcal{E}}_{\infty}$. According to the Bézout theorem, there are four base points, counted with multiplicities.

Proposition 7.1 The four base points are given by: 
- two base points of multiplicity 1 on each of the lines $\ell_{i}=0, i=1,2$ :

$$
B_{ \pm}^{(i)}=\left( \pm \frac{b_{i}}{\varepsilon d_{i j} d_{k i}}, \mp \frac{a_{i}}{\varepsilon d_{i j} d_{k i}}\right)
$$

The singular orbits of the map are as follows:

$$
\begin{aligned}
& \mathcal{L}_{-}^{(1)} \longrightarrow B_{-}^{(1)} \longrightarrow B_{+}^{(1)} \longrightarrow \mathcal{L}_{+}^{(1)}, \\
& \mathcal{L}_{-}^{(2)} \longrightarrow B_{-}^{(2)} \longrightarrow B_{+}^{(2)} \longrightarrow \mathcal{L}_{+}^{(2)},
\end{aligned}
$$

where $\mathcal{L}_{\mp}^{(i)}$ denotes the line through the points $B_{ \pm}^{(j)}, B_{ \pm}^{(k)}$.

Proof The singular orbits are a consequence of Proposition 3.2 and Theorem 3.3. It can be verified by straightforward computations that the points (49) are base points of the pencil of invariant curves $\overline{\mathcal{E}}_{\lambda}$.

With (25) we see that the point $B_{-}^{(3)}$ is a fixed point of $\phi_{+}$while $B_{+}^{(3)}$ is a fixed point of $\phi_{-}$. Therefore, they participate in patterns

$$
\begin{aligned}
\mathcal{L}_{-}^{(3)} \longrightarrow B_{-}^{(3)} \circlearrowleft & \\
\circlearrowright & B_{+}^{(3)} \longrightarrow \mathcal{L}_{+}^{(3)},
\end{aligned}
$$

which do not qualify as singularity confinement patterns $[15,21]$ and need not be blown up.

\subsection{Lifting the map to an analytically stable map}

We blow up the plane $\mathbb{C P}^{2}$ at the four base points $B_{-}^{(i)}, B_{+}^{(i)}, i=1,2$, and denote the corresponding exceptional divisors by $E_{i, 0}, E_{i, 1}, i=1,2$. The resulting blow-up surface is denoted by $X$. On this surface $\phi_{+}$is lifted to an analytically stable map $\widetilde{\phi}_{+}$ acting on the exceptional divisors according to the scheme [compare with (50)]

$$
\begin{aligned}
& \tilde{\mathcal{L}}_{-}^{(1)} \longrightarrow E_{1,0} \longrightarrow E_{1,1} \longrightarrow \tilde{\mathcal{L}}_{+}^{(1)}, \\
& \tilde{\mathcal{L}}_{-}^{(2)} \longrightarrow E_{2,0} \longrightarrow E_{2,1} \longrightarrow \widetilde{\mathcal{L}}_{+}^{(2)},
\end{aligned}
$$

where $\widetilde{\mathcal{L}}_{ \pm}^{(i)}$ denotes the proper transform of the line $\mathcal{L}_{ \pm}^{(i)}$.

We compute the induced pullback map on the Picard group $\widetilde{\phi}_{+}^{*}: \operatorname{Pic}(X) \rightarrow \operatorname{Pic}(X)$. Let $\mathcal{H} \in \operatorname{Pic}(X)$ be the pullback of the class of a generic line in $\mathbb{C P}^{2}$. Let $\varepsilon_{i, n} \in \operatorname{Pic}(X)$, for $i=1,2$ and $n=0,1$, be the class of $E_{i, n}$. Then the Picard group is

$$
\operatorname{Pic}(X)=\mathbb{Z} \mathcal{H} \bigoplus_{i=1}^{2} \bigoplus_{n=0}^{1} \mathbb{Z} \varepsilon_{i, n} .
$$




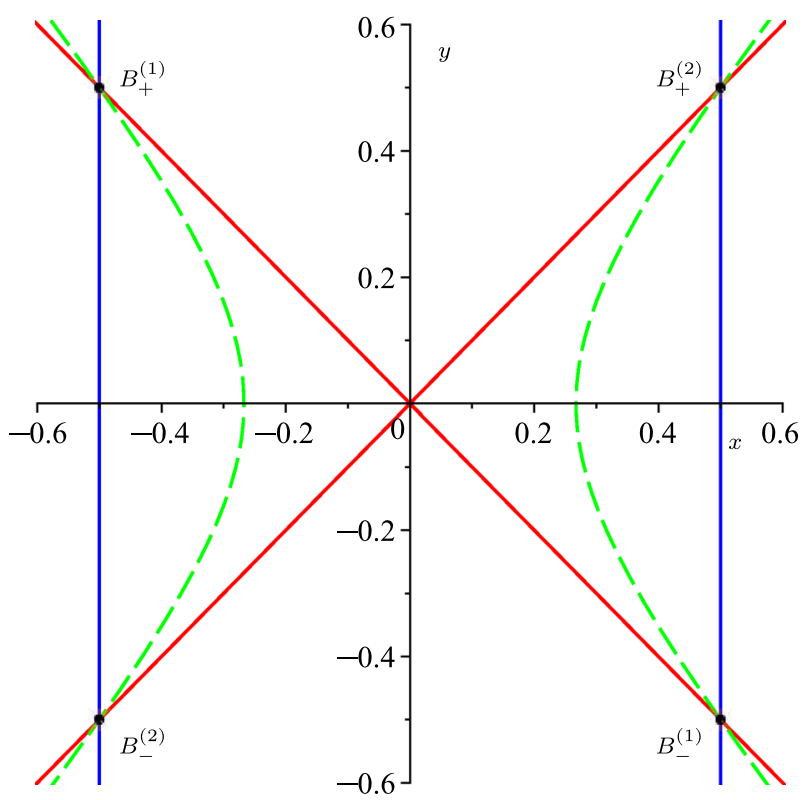

Fig. 4 The curves $\overline{\mathcal{E}}_{0}, \overline{\mathcal{E}}_{\infty}, \overline{\mathcal{E}}_{0.1}$ in resp. red, blue and green for $\ell_{1}(x, y)=x+y, \ell_{2}(x, y)=x-y$, $\ell_{3}(x, y)=x$ and $\varepsilon=1$ (Color figure online)

The rank of the Picard group is 5. The induced pullback $\widetilde{\phi}_{+}^{*}: \operatorname{Pic}(X) \rightarrow \operatorname{Pic}(X)$ is determined by (3).

With Theorem 2.3 we arrive at the system of recurrence relations for the degree $d(m)$ :

$$
\left\{\begin{array}{l}
d(m+1)=2 d(m)-\mu_{1}(m)-\mu_{2}(m), \\
\mu_{1}(m+2)=d(m)-\mu_{2}(m), \\
\mu_{2}(m+2)=d(m)-\mu_{1}(m),
\end{array}\right.
$$

with initial conditions $d(0)=1, \mu_{1}(m)=0$, for $m=0,1$, and $\mu_{2}(m)=0$, for $m=0,1$. The solution to this system of recurrence relations is given by

$$
\begin{aligned}
d(m) & =2 m, \\
\mu_{i}(m) & =m-1, \quad i=1,2 .
\end{aligned}
$$

The sequence $d(m)$ grows linearly.

8 The case $\left(\gamma_{1}, \gamma_{2}, \gamma_{3}\right)=(n, 1,-1)$

By Theorem 3.3 this case corresponds to the orbit data $\left(n_{1}, n_{2}\right)=(1, n),\left(\sigma_{1}, \sigma_{2}\right)=$ $(1,2)$. In this case, we consider the Kahan map $\phi_{+}: \mathbb{C}^{2} \rightarrow \mathbb{C}^{2}$ corresponding to a 
quadratic vector field of the form

$$
\dot{\mathbf{x}}=\frac{\ell_{3}^{2}(\mathbf{x})}{\ell_{1}^{n-1}(\mathbf{x})} J \nabla H(\mathbf{x}), \quad H(\mathbf{x})=\frac{\ell_{1}^{n}(\mathbf{x}) \ell_{2}(\mathbf{x})}{\ell_{3}(\mathbf{x})}
$$

The case $n=1$ was studied in [20].

For $\ell_{1}(\mathbf{x})=x, \ell_{2}(\mathbf{x})=x+y, \ell_{3}(\mathbf{x})=x-y$ the vector field reads

$$
\left\{\begin{array}{l}
\dot{x}=2 x^{2} \\
\dot{y}=-n x^{2}+n y^{2}+2 x y
\end{array}\right.
$$

and the Kahan discretization (13) reads

$$
\left\{\begin{array}{l}
\tilde{x}-x=4 \varepsilon \tilde{x} x, \\
\tilde{y}-y=2 \varepsilon(-n \tilde{x} x+n \tilde{y} y+\tilde{x} y+x \tilde{y}) .
\end{array}\right.
$$

Proposition 8.1 The Kahan map $\phi_{+}: \mathbb{C}^{2} \rightarrow \mathbb{C}^{2}$ admits an integral of motion

$$
\widetilde{H}(\mathbf{x})=\frac{H(\mathbf{x})}{P(\mathbf{x})},
$$

where

$$
P(\mathbf{x})=\prod_{k \in I}\left(\varepsilon d_{23} k \ell_{1}(\mathbf{x})+1\right)\left(\varepsilon d_{23} k \ell_{1}(\mathbf{x})-1\right)
$$

for $I=\{1,3,5, \ldots, n-1\}$ if $n$ is even and $I=\{2,4,6, \ldots, n-1\}$ if $n$ is odd.

Proof Note that the following identity holds:

$$
-d_{12} \ell_{3}(\mathbf{x})-d_{31} \ell_{2}(\mathbf{x})=d_{23} \ell_{1}(\mathbf{x}) .
$$

Then, using (52), from Eq. (14) it follows that

$$
\ell_{1}(\widetilde{\mathbf{x}})=\frac{\ell_{1}(\mathbf{x})}{2 \varepsilon d_{23} \ell_{1}(\mathbf{x})+1} .
$$

Moreover, multiplying (15) by $\ell_{3}(\mathbf{x})$ and (16) by $\ell_{2}(\mathbf{x})$ and then subtracting the second equation from the first equation and again applying (52), we arrive at

$$
\frac{\ell_{2}(\widetilde{\mathbf{x}})}{\ell_{3}(\widetilde{\mathbf{x}})}=-\frac{\ell_{2}(\mathbf{x})\left(\varepsilon d_{23}(n+1) \ell_{1}(\mathbf{x})+1\right)}{\ell_{3}(\mathbf{x})\left(\varepsilon d_{23}(n-1) \ell_{1}(\mathbf{x})-1\right)} .
$$

On the other hand, from (53) it follows that

$$
\varepsilon d_{23} k \ell_{1}(\widetilde{\mathbf{x}}) \pm 1=\frac{\varepsilon d_{23}(k \pm 2) \ell_{1}(\mathbf{x}) \pm 1}{2 \varepsilon d_{23} \ell_{1}(\mathbf{x})+1},
$$


and therefore, with $h_{ \pm}^{k}(\mathbf{x})=\left(\varepsilon d_{23} k \ell_{1}(\mathbf{x}) \pm 1\right)$, we find

$$
\begin{aligned}
\frac{P(\widetilde{\mathbf{x}})}{P(\mathbf{x})} & =\frac{h_{-}^{-1}(\mathbf{x}) h_{-}^{1}(\mathbf{x}) \cdots h_{-}^{n-3}(\mathbf{x}) \cdot h_{+}^{3}(\mathbf{x}) h_{+}^{5}(\mathbf{x}) \cdots h_{+}^{n+1}(\mathbf{x})}{\left(h_{+}^{2}(\mathbf{x})\right)^{n} \cdot h_{-}^{1}(\mathbf{x}) h_{-}^{3}(\mathbf{x}) \cdots h_{-}^{n-1}(\mathbf{x}) \cdot h_{+}^{1}(\mathbf{x}) h_{+}^{3}(\mathbf{x}) \cdots h_{+}^{n-1}(\mathbf{x})} \\
& =-\frac{h_{+}^{n+1}(\mathbf{x})}{\left(h_{+}^{2}(\mathbf{x})\right)^{n} h_{-}^{n-1}(\mathbf{x})},
\end{aligned}
$$

if $n$ is even, and

$$
\begin{aligned}
\frac{P(\widetilde{\mathbf{x}})}{P(\mathbf{x})} & =\frac{h_{-}^{0}(\mathbf{x}) h_{-}^{2}(\mathbf{x}) \cdots h_{-}^{n-3}(\mathbf{x}) \cdot h_{+}^{4}(\mathbf{x}) h_{+}^{6}(\mathbf{x}) \cdots h_{+}^{n+1}(\mathbf{x})}{\left(h_{+}^{2}(\mathbf{x})\right)^{n-1} \cdot h_{-}^{2}(\mathbf{x}) h_{-}^{4}(\mathbf{x}) \cdots h_{-}^{n-1}(\mathbf{x}) \cdot h_{+}^{2}(\mathbf{x}) h_{+}^{4}(\mathbf{x}) \cdots h_{+}^{n-1}(\mathbf{x})} \\
& =-\frac{h_{+}^{n+1}(\mathbf{x})}{\left(h_{+}^{2}(\mathbf{x})\right)^{n} h_{-}^{n-1}(\mathbf{x})},
\end{aligned}
$$

if $n$ is odd. This proves the claim.

With Theorem 2.3 we arrive at the system of recurrence relations for the degree $d(m)$ :

$$
\left\{\begin{array}{l}
d(m+1)=2 d(m)-\mu_{1}(m)-\mu_{2}(m) \\
\mu_{1}(m+1)=d(m)-\mu_{2}(m) \\
\mu_{2}(m+n)=d(m)-\mu_{1}(m)
\end{array}\right.
$$

with initial conditions $d(0)=1, \mu_{1}(0)=0$ and $\mu_{2}(m)=0$, for $m=0, \ldots, n-1$. The generating functions of the solution to this system of recurrence relations are given by

$$
\begin{aligned}
d(z) & =1+2 z+\cdots+n z^{n-1}+\frac{(n+1) z^{n}}{1-z}, \\
\mu_{1}(z) & =z+2 z^{2}+\cdots+(n-1) z^{n-1}+\frac{n z^{n}}{1-z}, \\
\mu_{2}(z) & =\frac{z^{n}}{1-z}
\end{aligned}
$$

Note that the degrees of $\phi_{+}^{k}$ grow linearly for $k=1, \ldots, n-1$ and stabilize to $n+1$ for $k \geqslant n$. This seems to be the first example of a birational map of deg $=2$ with such behavior.

Acknowledgements The author would like to thank Matteo Petrera and Yuri Suris for their critical feedback on this manuscript.

Funding Open Access funding enabled and organized by Projekt DEAL.

Open Access This article is licensed under a Creative Commons Attribution 4.0 International License, which permits use, sharing, adaptation, distribution and reproduction in any medium or format, as long as you give appropriate credit to the original author(s) and the source, provide a link to the Creative Commons licence, and indicate if changes were made. The images or other third party material in this article are included 
in the article's Creative Commons licence, unless indicated otherwise in a credit line to the material. If material is not included in the article's Creative Commons licence and your intended use is not permitted by statutory regulation or exceeds the permitted use, you will need to obtain permission directly from the copyright holder. To view a copy of this licence, visit http://creativecommons.org/licenses/by/4.0/.

\section{References}

1. Bedford, E., Kim, K.: On the degree growth of birational mappings in higher dimensions. J. Geom. Anal. 14(4), 567-596 (2004)

2. Bedford, E., Kim, K.: Periodicities in linear fractional recurrences: degree growth of birational surface maps. Michigan Math. J. 54(3), 647-670 (2006)

3. Carstea, A.S., Takenawa, T.: A note on minimization of rational surfaces obtained from birational dynamical systems. J. Nonlinear Math. Phys. 20(suppl. 1), 17-33 (2013)

4. Celledoni, E., McLachlan, R.I., Owren, B., Quispel, G.R.W.: Geometric properties of Kahan's method. J. Phys. A 46(2), 12 pp. (2013)

5. Celledoni, E., McLachlan, R.I., McLaren, D.I., Owren, B., Quispel, G.R.W.: Integrability properties of Kahan's method. J. Phys. A 47(36), 20 pp. (2014)

6. Celledoni, E., McLachlan, R.I., McLaren, D.I., Owren, B., Quispel, G.R.W.: Two classes of quadratic vector fields for which the Kahan map is integrable. MI Lecture Notes, Kyushu University 74, pp. 60-62 (2016). arXiv:1702.00280

7. Cerveau, D., Déserti, J.: Transformations Birationnelles de Petit Degré. Cours Spécialisés, vol. 19. Société Mathématique de France, Paris (2013)

8. Diller, J.: Cremona transformations, surface automorphisms, and plane cubics. Michigan Math. J. 60(2), 409-440 (2011)

9. Diller, J., Favre, C.: Dynamics of bimeromorphic maps of surfaces. Amer. J. Math. 123(6), 1135-1169 (2001)

10. Hartshorne, R.: Algebraic Geometry. Graduate Texts in Mathematics, vol. 52. Springer, New York (1977)

11. Hitchin, N.J., Manton, N.S., Murray, M.K.: Symmetric monopoles. Nonlinearity 8(5), 661-692 (1995)

12. Hone, A.N.W., Petrera, M.: Three-dimensional discrete systems of Hirota-Kimura type and deformed Lie-Poisson algebras. J. Geom. Mech. 1(1), 55-85 (2009)

13. Kahan, W.: Unconventional numerical methods for trajectory calculations. Unpublished lecture notes (1993)

14. van der Kamp, P.H., Celledoni, E., McLachlan, R.I., McLaren, D.I., Owren, B., Quispel, G.R.W.: Three classes of quadratic vector fields for which the Kahan discretization is the root of a generalised Manin transformation. J. Phys. A 52(4), 10 pp. (2019)

15. Mase, T., Willox, R., Ramani, A., Grammaticos, B.: Singularity confinement as an integrability criterion. J. Phys. A 52(20), 29 pp. (2019)

16. Petrera, M., Pfadler, A., Suris, Yu.B.: On integrability of Hirota-Kimura-type discretizations: Experimental study of the discrete Clebsch system. Experiment. Math. 18(2), 223-247 (2009)

17. Petrera, M., Pfadler, A., Suris, Yu.B.: On integrability of Hirota-Kimura type discretizations. Regul. Chaotic Dyn. 16(3-4), 245-289 (2011)

18. Petrera, M., Pfadler, A., Suris, Yu.B.: Geometry of the Kahan discretizations of planar quadratic Hamiltonian systems. Proc. A. 475(2223), 13 pp. (2019)

19. Petrera, M., Suris, Yu.B.: Geometry of the Kahan discretizations of planar quadratic Hamiltonian systems. II. Systems with a linear Poisson tensor. J. Comput. Dyn. 6(2), 401-408 (2019)

20. Petrera, M., Zander, R.: New classes of quadratic vector fields admitting integral-preserving KahanHirota-Kimura discretizations. J. Phys. A 50(20), Art. No. 205203 (2017)

21. Takenawa, T., Eguchi, M., Grammaticos, B., Otha, Y., Ramani, A., Satsuma, J.: The space of initial conditions for linearizable mappings. Nonlinearity 16(2), 457-477 (2003)

Publisher's Note Springer Nature remains neutral with regard to jurisdictional claims in published maps and institutional affiliations. 